\title{
DRAWDOWN AND DRAWUP FOR FRACTIONAL BROWNIAN MOTION WITH TREND
}

\author{
LONG BAI AND PENG LIU
}

\begin{abstract}
We consider the drawdown and drawup of a fractional Brownian motion with trend, which corresponds to the logarithm of geometric fractional Brownian motion representing the stock price in financial market. We derive the asymptotics of tail probabilities of the maximum drawdown and maximum drawup as the threshold goes to infinity, respectively. It turns out that the extremes of drawdown leads to new scenarios of asymptotics depending on Hurst index of fractional Brownian motion.
\end{abstract}

Key Words: Drawdown; Drawup; Fractional Brownian motion; Geometric fractional Brownian motion; Pickands constant; Piterbarg constant.

AMS Classification: Primary 60G15; secondary 60G70

\section{Introduction ANd PReliminaries}

Drawdown, defined as the distance of the present value away from its historical running maximum, is an important indicator of downside risks in financial risk management. For instance, the drawdown and the maximum drawdown have been customarily used as risk measures in finance where they measure the current drop of a stock price, an index or the value of a portfolio from its running maximum; see, e.g., [1, 2]. Instead of Value-at-Risk, the Maximum Drawdown-at-Risk has been proposed to capture the cumulative losses; see [3]. Moreover, maximum drawdown and maximum drawup also appear in the portfolio sensitivities of underlying asset; see [4]. They can also be deployed in the context of portfolio optimization as constrains; see, e.g., [5, 6]. Drawdown processes also appear in other applications, such as applied probability and queueing theory; see, e.g., [7-10]. Complementary, drawup, the dual of drawdown, which is the distance of current value from its historical running minimum, has been encountered in many financial applications; see, e.g., [11, 2].

In the literature, e.g., $[12,13]$, the stock price $S$ can be modeled by the so-called geometric fractional Brownian motion, i.e.,

$$
S_{t}=S_{0} \exp \left(\mu t+\sigma B_{H}(t)-\frac{1}{2} \sigma^{2} t^{2 H}\right)
$$

where $\sigma>0, \mu \in \mathbb{R}$ and $B_{H}$ is a fractional Brownian motion (fBm) with Hurst index $H \in(0,1)$ and covariance function satisfying

$$
\operatorname{Cov}\left(B_{H}(s), B_{H}(t)\right)=\frac{|s|^{2 H}+|t|^{2 H}-|s-t|^{2 H}}{2}, s, t \geq 0 .
$$

Note that $S_{t}$ is reduced to geometric Brownian motion if $H=1 / 2$ which has massive applications in finance. To facilitate our analysis, we shall work with the log-prices. This motivates us to consider the drawdown and drawup for fBm with trend. Let $X_{t}=\mu t+\sigma B_{H}(t)-\frac{1}{2} \sigma^{2} t^{2 H}, \mu \in \mathbb{R}$. Without of loss of generality, we assume that $\sigma=1$. The drawdown and drawup processes of $X$ are defined, respectively, by

$$
D_{t}=\bar{X}_{t}-X_{t}, \quad U_{t}=X_{t}-\underline{X}_{t}
$$

where $\bar{X}_{t}=\sup _{0 \leq s \leq t} X_{s}$ and $\underline{X}_{t}=\inf _{0 \leq s \leq t} X_{s}$. For some fixed $T \in(0, \infty)$, we are interested in, for any $u>0$,

$$
\mathbb{P}\left\{\sup _{0 \leq t \leq T} D_{t}>u\right\} \quad \text { and } \mathbb{P}\left\{\sup _{0 \leq t \leq T} U_{t}>u\right\} .
$$

Date: May 20, 2018. 
Notice that the maximum of drawdown over $[0, T]$ has the interpretation as the largest log-loss up to time $T$ and accordingly, the maximum of drawup can be viewed as the largest log-return; see e.g., [9]. Additionally, for $H=\frac{1}{2}$, in the context of queueing theory, $D_{t}$ is the transient queue length process starting at 0 and the corresponding probability in (2) represents the overload probability over $[0, T]$; see, e.g., [7, 8].

Note that for the special case $H=1 / 2$, the exact expressions of (2) were obtained in [14, 15]; see also [16] concerning the joint distribution of maximum drawdown and maximum drawup up to an independent exponential time. Due to the fact that $\mathrm{fBm}$ is neither a semi-martingale nor a Markov process, the exact expressions for $H \neq \frac{1}{2}$ are not available in the literature. Hence in this paper we focus on the asymptotics of $(2)$ as $u \rightarrow \infty$.

It is worthwhile to mention that infinite series representation of $(2)$ in $[14,15]$ for $H=\frac{1}{2}$ is quite complicated. In contrast, we get concise asymptotics for $H=1 / 2$ in this paper. Theorems 2.1 and 2.2 in Section 2 show that, for $H=\frac{1}{2}$, as $u \rightarrow \infty$,

$$
\mathbb{P}\left\{\sup _{0 \leq t \leq T} D_{t}>u\right\} \sim 4 \mathbb{P}\left\{\mathcal{N}>\frac{u+\left(\mu-\frac{1}{2} T\right)}{\sqrt{T}}\right\}, \quad \mathbb{P}\left\{\sup _{0 \leq t \leq T} U_{t}>u\right\} \sim 4 \mathbb{P}\left\{\mathcal{N}>\frac{u+\left(\frac{1}{2} T-\mu\right)}{\sqrt{T}}\right\},
$$

where $\mathcal{N}$ is a standard normal random variable and $\sim$ means asymptotic equivalence when the arguments go to 0 or infinity.

The technique used in this paper is uniform double-sum method in [17], which is the development of the so-called double-sum method widely applied in extreme value theory of Gaussian processes and random fields; see, e.g., [18]. As it is shown in Theorem 2.1 in Section 2, the special trend renders the asymptotics for drawdown quite different from those of non-centered Gaussian random fields related to fBm in the literature (see, e.g., [19-22]), leading to new scenarios of asymptotics for some $H$.

Our results can be applied to calculate the Maximum Drawdown-at-Risk and the probability of stock market crashes and rallies for (1); see [3] and [1].

We next introduce some useful notation. We begin with Pickands constant, which is defined by

$$
\mathcal{H}_{H}=\lim _{b \rightarrow \infty} \frac{1}{b} \mathcal{H}_{H}([0, b]) \quad \text { with } \mathcal{H}_{H}([a, b])=\mathbb{E}\left\{\sup _{t \in[a, b]} e^{\sqrt{2} B_{H}(t)-|t|^{2 H}}\right\}, \quad a<b .
$$

Further, Piterbarg constant is given by, for $\nu>0$,

$$
\mathcal{P}_{H}^{\nu}=\lim _{b \rightarrow \infty} \mathcal{P}_{H}^{\nu}([0, b]) \quad \text { with } \mathcal{P}_{H}^{\nu}([0, b])=\mathbb{E}\left\{\sup _{t \in[0, b]} e^{\sqrt{2} B_{H}(t)-(1+\nu)|t|^{2 H}}\right\}, \quad b>0 .
$$

We refer to [18, 23-27] for the definition, properties and extensions of Pickands and Piterbarg constants, and to [28-32] for the bounds and simulations of Pickands and Piterbarg constants. In particular, by [28], we have that

$$
\mathcal{P}_{1 / 2}^{\nu}=1+\frac{1}{\nu}, \quad \nu>0
$$

Our paper is organized as follows. In Section 2, we establish our main theorems. The proofs of these results are given in Section 3. Proofs of some technical lemmas are postponed in Appendix A, followed by some useful lemmas in Appendix B.

\section{MAin Results}

In this section, we present our main results concerning the asymptotics of (2) as $u \rightarrow \infty$. In contrast to the infinite series representation in $[14,15]$, the asymptotic expressions in the following theorems are quite concise, which allows us to readily understand the asymptotic behavior of the probability that the maximum drawdown ( maximum drawup) exceeds a threshold over finite-time horizon. Let $\Psi(u):=\mathbb{P}\{\mathcal{N}>u\}$. Then we have the following results. 
Theorem 2.1. Assume that $0<T<\infty$.

If $H>1 / 2$, then

$$
\mathbb{P}\left\{\sup _{0 \leq t \leq T} D_{t}>u\right\} \sim \Psi\left(\frac{u+\mu T-\frac{1}{2} T^{2 H}}{T^{H}}\right)
$$

If $H=1 / 2$, then

$$
\mathbb{P}\left\{\sup _{0 \leq t \leq T} D_{t}>u\right\} \sim 4 \Psi\left(\frac{u+\mu T-\frac{1}{2} T}{T^{\frac{1}{2}}}\right)
$$

If $1 / 4<H<1 / 2$, then

$$
\mathbb{P}\left\{\sup _{0 \leq t \leq T} D_{t}>u\right\} \sim\left(H^{-1} 2^{-\frac{1}{2 H}} T^{2 H-1} \mathcal{H}_{H}\right)^{2} u^{\frac{2}{H}-4} \Psi\left(\frac{u+\mu T-\frac{1}{2} T^{2 H}}{T^{H}}\right) .
$$

If $H=1 / 4$, then

$$
\mathbb{P}\left\{\sup _{0 \leq t \leq T} D_{t}>u\right\} \sim\left(\mathcal{H}_{\frac{1}{4}}\right)^{2} T^{-1} \int_{0}^{\infty} e^{-x-T^{\frac{1}{4}} x^{\frac{1}{2}}} d x u^{4} \Psi\left(\frac{u+\mu T-\frac{1}{2} T^{\frac{1}{2}}}{T^{\frac{1}{4}}}\right) .
$$

If $0<H<1 / 4$, then

$$
\mathbb{P}\left\{\sup _{0 \leq t \leq T} D_{t}>u\right\} \sim H^{-1} 2^{-\frac{1}{2 H}} T^{2 H-2} \Gamma\left(\frac{1}{2 H}+1\right)\left(\mathcal{H}_{H}\right)^{2} u^{\frac{3}{2 H}-2} \Psi\left(\frac{u+\mu T-\frac{1}{2} T^{2 H}}{T^{H}}\right) .
$$

Theorem 2.2. Assume that $0<T<\infty$.

If $H>1 / 2$, then

$$
\mathbb{P}\left\{\sup _{0 \leq t \leq T} U_{t}>u\right\} \sim \Psi\left(\frac{u-\mu T+\frac{1}{2} T^{2 H}}{T^{H}}\right)
$$

If $H=1 / 2$, then

$$
\mathbb{P}\left\{\sup _{0 \leq t \leq T} U_{t}>u\right\} \sim 4 \Psi\left(\frac{u-\mu T+\frac{1}{2} T}{T^{\frac{1}{2}}}\right) .
$$

If $0<H<1 / 2$, then

$$
\mathbb{P}\left\{\sup _{0 \leq t \leq T} U_{t}>u\right\} \sim 2^{-\frac{1}{H}-\frac{1}{2}} T^{3 H} \sqrt{\frac{\pi}{H^{3}(H-1)}}\left(\mathcal{H}_{H}\right)^{2} u^{\frac{2}{H}-3} \Psi\left(\inf _{0 \leq s \leq T} \frac{u-\mu(T-s)+\frac{1}{2}\left(T^{2 H}-s^{2 H}\right)}{(T-s)^{H}}\right) .
$$

Remark 2.3. i) In the extremes of Gaussian processes and random fields associated with fBm over finitetime horizon, e.g.,[19-22], one usually has three different types of asymptotics depending on the fact that $H>$ $1 / 2, H=1 / 2$ and $H<1 / 2$. However, Theorem 2.1 gives more types of asymptotics due to the complexity of the trend that is the combination of linear function $(\mu t)$ and power function $\left(-\frac{1}{2}|t|^{2 H}\right)$. As we can see from the proof of Theorem 2.1, for $1 / 4<H<1 / 2$ only the linear part of trend contributes to the power part of the asymptotics; for $H=1 / 4$, both linear and power parts of the trend affect the power part of the asymptotics; whereas, for $0<H<1 / 4$, the power part of the trend has the major influence on the power part of the asymptotics. However, this phenomena does not appear in Theorem 2.2, where both of linear trend and power trend contribute to the power part of the asymptotics for $0<H<1 / 2$.

ii) We here interpret that the analysis of drawdown and drawup for the case $T=\infty$ is meaningless. Let $T=\infty$ and $\widetilde{B}_{H}=-B_{H}$. Then

$$
\begin{aligned}
\sup _{0 \leq t<\infty} D_{t} & =\sup _{0 \leq s \leq t<\infty}\left(B_{H}(s)-B_{H}(t)+\frac{1}{2}\left(t^{2 H}-s^{2 H}\right)-\mu(t-s)\right) \\
& =\sup _{0 \leq s \leq t<\infty}\left(\widetilde{B}_{H}(t)-\widetilde{B}_{H}(s)+\frac{1}{2}\left(t^{2 H}-s^{2 H}\right)-\mu(t-s)\right) \\
& \geq \sup _{0 \leq s \leq t<\infty}\left(\widetilde{B}_{H}(t)-\widetilde{B}_{H}(s)-(|\mu|+1)(t-s)\right) \\
& =\sup _{s \geq 0} Q(s)
\end{aligned}
$$


where

$$
Q(s)=\sup _{t \geq s}\left(\widetilde{B}_{H}(t)-\widetilde{B}_{H}(s)-(|\mu|+1)(t-s)\right) .
$$

Corollary 1 in [33] (see also Corollary 1 in [34]) shows that for $H \in(0,1)$

$$
\limsup _{s \rightarrow \infty} \frac{Q(s)}{(\log s)^{\frac{1}{2(1-H)}}}=C>0 \quad \text { a.s.. }
$$

Therefore we have that for $H \in(0,1)$

$$
\sup _{0 \leq t<\infty} D_{t} \geq \sup _{s \geq 0} Q(s)=\infty \quad \text { a.s.. }
$$

Note that for $t \geq s \geq 1$ and $H \in(0,1 / 2]$, there exists $C_{1}>0$ such that

$$
t^{2 H}-s^{2 H} \leq C_{1}(t-s)
$$

Hence for $H \in(0,1 / 2]$

$$
\begin{aligned}
\sup _{0 \leq t<\infty} U_{t} & =\sup _{0 \leq s \leq t<\infty}\left(B_{H}(t)-B_{H}(s)-\frac{1}{2}\left(t^{2 H}-s^{2 H}\right)+\mu(t-s)\right) \\
& \geq \sup _{1 \leq s \leq t<\infty}\left(B_{H}(t)-B_{H}(s)-C_{2}(t-s)\right)=\infty \quad \text { a.s., }
\end{aligned}
$$

where $C_{2}$ is a positive constant. We can also show that for $H>1 / 2$

$$
\sup _{0 \leq t<\infty} U_{t}=\infty \quad \text { a.s. }
$$

which needs more technical analysis similarly to [33, 34].

\section{ProOFS}

In this section we give the proof of Theorems 2.1-2.2. In order to prove the aforementioned theorems, we first present several lemmas related to the local behavior of variance and correlation functions of the underlying Gaussian random fields. In the rest of the paper, denote by $Q, Q_{i}, i=1,2, \ldots$ some positive constants that may differ from line to line.

Let

$$
\sigma_{u}^{ \pm}(s, t)=\frac{\sigma_{H}(s, t)}{u \mp \mu(t-s) \pm \frac{1}{2}\left(t^{2 H}-s^{2 H}\right)}, \quad(s, t) \in A:=\{(s, t): 0 \leq s \leq t \leq T\}
$$

where

$$
\sigma_{H}(s, t):=\sqrt{\operatorname{Var}\left(B_{H}(t)-B_{H}(s)\right)}=|t-s|^{H}
$$

Lemma 3.1. For any $H \in(0,1)$ and $u$ large enough, $\arg \sup _{0 \leq s \leq t \leq T} \sigma_{u}^{-}(s, t)$ is unique and equals $(0, T)$. Moreover for any function $\delta_{u}$ such that $\delta_{u}>0$ and $\lim _{u \rightarrow \infty} \delta_{u}=0$, we have

$$
\lim _{u \rightarrow \infty} \sup _{(s, t) \neq(0, T),(s, t) \in\left[0, \delta_{u}\right] \times\left[T-\delta_{u}, T\right]}\left|\frac{1-\frac{\sigma_{u}^{-}(s, t)}{\sigma_{u}^{-}(0, T)}}{\frac{H(T-t)}{T}+\frac{H}{T} s+\frac{1}{2 u} s^{2 H}}-1\right|=0 .
$$

Lemma 3.2. i) For $H \geq \frac{1}{2}$ and u large enough, $\arg \sup _{0 \leq s \leq t \leq T} \sigma_{u}^{+}(s, t)$ is unique and equals $(0, T)$. Moreover, for any function $\delta_{u}$ such that $\delta_{u}>0$ and $\lim _{u \rightarrow \infty} \delta_{u}=0$, we have

$$
\lim _{u \rightarrow \infty} \sup _{(s, t) \neq(0, T),(s, t) \in\left[0, \delta_{u}\right] \times\left[T-\delta_{u}, T\right]}\left|\frac{1-\frac{\sigma_{u}^{+}(s, t)}{\sigma_{u}^{+}(0, T)}}{\frac{H(T-t)}{T}+\frac{H}{T} s}-1\right|=0 .
$$


ii) For $0<H<\frac{1}{2}$ and $u$ large enough, $\arg \sup _{0 \leq s \leq t \leq T} \sigma_{u}^{+}(s, t)$ is unique and equals $\left(s_{u}, T\right)$ with $s_{u} \sim$ $T^{\frac{1}{1-2 H}} u^{-\frac{1}{1-2 H}}, u \rightarrow \infty$. Moreover, for any $\delta_{u}$ such that $\delta_{u}>0$ and $\lim _{u \rightarrow \infty} \delta_{u}=0$, we have

$$
\lim _{u \rightarrow \infty} \sup _{(s, t) \neq\left(s_{u}, T\right),(s, t) \in\left[0, s_{u}+\delta_{u}\right] \times\left[T-\delta_{u}, T\right]}\left|\frac{1-\frac{\sigma_{u}^{+}(s, t)}{\sigma_{u}^{+}\left(s_{u}, T\right)}}{\frac{H(T-t)}{T}+\frac{H(1-H)}{2 T^{2}}\left(s-s_{u}\right)^{2}}-1\right|=0 .
$$

Lemma 3.3. For any function $\delta_{u}$ such that $\delta_{u}>0$ and $\lim _{u \rightarrow \infty} \delta_{u}=0$, we have

$$
\lim _{u \rightarrow \infty} \sup _{(s, t) \neq\left(s^{\prime}, t^{\prime}\right),(s, t),\left(s^{\prime}, t^{\prime}\right) \in\left[0, \delta_{u}\right] \times\left[T-\delta_{u}, T\right]}\left|\frac{1-\operatorname{Corr}\left(B_{H}(t)-B_{H}(s), B_{H}\left(t^{\prime}\right)-B_{H}\left(s^{\prime}\right)\right)}{\left|s-s^{\prime}\right|^{2 H}+\left|t-t^{\prime}\right|^{2 H}} \times 2 T^{2 H}-1\right|=0 .
$$

Proof of Theorem 2.1: Observe that

$$
\begin{aligned}
\mathbb{P}\left\{\sup _{0 \leq t \leq T} D_{t}>u\right\} & =\mathbb{P}\left\{\sup _{(s, t) \in A}\left(X_{s}-X_{t}\right)>u\right\} \\
& =\mathbb{P}\left\{\sup _{(s, t) \in A}\left(B_{H}(s)-B_{H}(t)+\mu(s-t)-\frac{1}{2}\left(s^{2 H}-t^{2 H}\right)\right)>u\right\} \\
& =\mathbb{P}\left\{\sup _{(s, t) \in A} Z_{u}(s, t)>m(u)\right\},
\end{aligned}
$$

where

$$
Z_{u}(s, t)=\frac{B_{H}(s)-B_{H}(t)}{u+\mu(t-s)+\frac{1}{2}\left(s^{2 H}-t^{2 H}\right)} m(u), \quad m(u)=\frac{u+\mu T-\frac{1}{2} T^{2 H}}{T^{H}} .
$$

In the first step of the proof, we subdivide the set $A$ into two subsets:

$$
E_{u}=\left[0,(\ln m(u))^{2} / m^{2}(u)\right] \times\left[T-(\ln m(u))^{2} / m^{2}(u), T\right],
$$

the neighborhood of the point $(0, T)$, which maximizes the variance of $Z_{u}(s, t)$ (with high probability the supremum is realized in $\left.E_{u}\right)$ and the set $A \backslash E_{u}$, over which the probability associated with supremum is asymptotically negligible. Then we have

$$
\begin{aligned}
\mathbb{P}\left\{\sup _{(s, t) \in E_{u}} Z_{u}(s, t)>m(u)\right\} & \leq \mathbb{P}\left\{\sup _{0 \leq t \leq T} D_{t}>u\right\} \\
& \leq \mathbb{P}\left\{\sup _{(s, t) \in E_{u}} Z_{u}(s, t)>m(u)\right\}+\mathbb{P}\left\{\sup _{(s, t) \in A \backslash E_{u}} Z_{u}(s, t)>m(u)\right\} .
\end{aligned}
$$

In light of Lemma 3.1, it follows that for $u$ sufficiently large, $\sqrt{\operatorname{Var}\left(Z_{u}(s, t)\right)}=\frac{\sigma_{u}^{-}(s, t)}{\sigma_{u}^{-}(0, T)}$ attains its maximum over $0 \leq s \leq t \leq T$ at a unique point, with coordinates $(0, T)$. Moreover, there exists a positive constant $Q$ such that

$$
\begin{aligned}
\sup _{(s, t) \in A \backslash E_{u}} \sqrt{\operatorname{Var}\left(Z_{u}(s, t)\right)} & \leq 1-\frac{1}{2} \inf _{(s, t) \in A \backslash E_{u}}\left(\frac{H(T-t)}{T}+\frac{H}{T} s+\frac{1}{2 u} s^{2 H}\right) \\
& \leq 1-\frac{1}{2} \inf _{(s, t) \in A \backslash E_{u}}\left(\frac{H(T-t)}{T}+\frac{H}{T} s\right) \\
& \leq 1-Q\left(\frac{\ln m(u)}{m(u)}\right)^{2} .
\end{aligned}
$$

Notice that the length of $E_{u}$ on each coordinates being $\left(\frac{\ln m(u)}{m(u)}\right)^{2}$ guarantees that the last inequality above holds. Moreover,

$$
\mathbb{E}\left(\left(Z_{u}(s, t)-Z_{u}\left(s^{\prime}, t^{\prime}\right)\right)^{2}\right) \leq Q_{1}\left(\left|s-s^{\prime}\right|^{2 H}+\left|t-s^{\prime}\right|^{2 H}\right), \quad(s, t),\left(s^{\prime}, t^{\prime}\right) \in A,
$$


with $Q_{1}$ a positive constant. Hence by Piterbarg Theorem (Theorem 8.1 in [18]), we have for $u$ sufficiently large

$$
\mathbb{P}\left\{\sup _{(s, t) \in A \backslash E_{u}} Z_{u}(s, t)>m(u)\right\} \leq Q_{2}(m(u))^{\frac{2}{H}} \Psi\left(\frac{m(u)}{1-Q\left(\frac{\ln m(u)}{m(u)}\right)^{2}}\right) .
$$

Next we analyze $\mathbb{P}\left\{\sup _{(s, t) \in E_{u}} Z_{u}(s, t)>u\right\}$. Let

$$
\Delta(u)=2^{\frac{1}{2 H}} T(m(u))^{-\frac{1}{H}}, \quad E_{u, 1}=\left[0,(\ln m(u))^{2} /\left(m^{2}(u) \Delta(u)\right)\right]^{2} .
$$

Then rewrite

$$
\mathbb{P}\left\{\sup _{(s, t) \in E_{u}} Z_{u}(s, t)>u\right\}=\mathbb{P}\left\{\sup _{(s, t) \in E_{u, 1}} Z_{u}(\Delta(u) s, T-\Delta(u) t)>u\right\} .
$$

We distinguish between $H>\frac{1}{2}, H=\frac{1}{2}, \frac{1}{4}<H<\frac{1}{2}, H=\frac{1}{4}$ and $0<H<\frac{1}{4}$.

Case $H>\frac{1}{2}$. In order to apply Lemma 4.1 in Appendix, we need to check conditions. By Lemmas 3.1 and 3.3, we have

$$
\lim _{u \rightarrow \infty} \sup _{(s, t) \neq(0,0),(s, t) \in E_{u, 1}}\left|\frac{1-\sqrt{\operatorname{Var}\left(Z_{u}(\Delta(u) s, T-\Delta(u) t)\right)}}{\Delta(u)\left(\frac{H}{T} t+\frac{H}{T} s\right)}-1\right|=0,
$$

and

$$
\lim _{u \rightarrow \infty} \sup _{(s, t) \neq\left(s^{\prime}, t^{\prime}\right),(s, t),\left(s^{\prime}, t^{\prime}\right) \in E_{u, 1}}\left|m^{2}(u) \frac{1-\operatorname{Corr}\left(Z_{u}(\Delta(u) s, T-\Delta(u) t), Z_{u}\left(\Delta(u) s^{\prime}, T-\Delta(u) t^{\prime}\right)\right)}{\left|s-s^{\prime}\right|^{2 H}+\left|t-t^{\prime}\right|^{2 H}}-1\right|=0 .
$$

This implies that (27) and (28) hold. Following the notation in Lemma 4.1, we have for $H>\frac{1}{2}$

$$
\nu_{i}=\lim _{u \rightarrow \infty}(m(u))^{2} \frac{H}{T} \Delta(u)=\lim _{u \rightarrow \infty} 2^{\frac{1}{2 H}} H T^{1-2 H} u^{2-\frac{1}{H}}=\infty, i=1,2 .
$$

Noting that $(0,0) \in E_{u, 1}$ and by case iii) of Lemma 4.1 in Appendix, we have

$$
\mathbb{P}\left\{\sup _{(s, t) \in E_{u, 1}} Z_{u}(\Delta(u) s, T-\Delta(u) t)>m(u)\right\} \sim \Psi(m(u)),
$$

which together with (4) and (5) establishes the claim.

Case $H=\frac{1}{2}$. Note that (6) and (7) still hold for $H=\frac{1}{2}$. Following the notation in Lemma 4.1, we have for $H=\frac{1}{2}$ and $i=1,2$,

$$
\nu_{i}=\lim _{u \rightarrow \infty}(m(u))^{2} \frac{H}{T} \Delta(u)=2^{\frac{1}{2 H}} H=1, \quad \lim _{u \rightarrow \infty} a_{i}(u)=0, \quad \lim _{u \rightarrow \infty} b_{i}(u)=\lim _{u \rightarrow \infty}(\ln m(u))^{2} /\left(m^{2}(u) \Delta(u)\right)=\infty .
$$

Thus by case ii) of Lemma 4.1 in Appendix, we have

$$
\mathbb{P}\left\{\sup _{(s, t) \in E_{u, 1}} Z_{u}(\Delta(u) s, T-\Delta(u) t)>m(u)\right\} \sim\left(\mathcal{P}_{1 / 2}^{1}\right)^{2} \Psi(m(u)),
$$

which combined with (4), (5) and (3) establishes the claim.

Case $\frac{1}{4}<H<\frac{1}{2}$. The idea of the proof: we first divide $E_{u, 1}$ into many sub-rectangles (the Pickands rectangles) and derive the uniform asymptotics on each rectangle; then with the aid of Bonferroni inequality we show that the double-sum term is negligible and the asymptotics over $E_{u, 1}$ is the summation of the asymptotics on these rectangles. For any fixed $S$, let

$$
\begin{gathered}
I_{k, l}=[k S,(k+1) S] \times[l S,(l+1) S], k, l \geq 0, \quad N(u)=\left[\frac{(\ln m(u))^{2}}{m^{2}(u) \Delta(u) S}\right], \\
\Lambda_{1}(u)=\left\{\left(k, l, k^{\prime}, l^{\prime}\right): 0 \leq k, l, k^{\prime}, l^{\prime} \leq N(u)+1, I_{k, l} \cap I_{k^{\prime}, l^{\prime}} \neq \emptyset,(k, l) \neq\left(k^{\prime}, l^{\prime}\right)\right\}, \\
\Lambda_{2}(u)=\left\{\left(k, l, k^{\prime}, l^{\prime}\right): 0 \leq k, l, k^{\prime}, l^{\prime} \leq N(u)+1, I_{k, l} \cap I_{k^{\prime}, l^{\prime}}=\emptyset\right\} .
\end{gathered}
$$


Note that in the following proof, we may let $S \rightarrow \infty$. Bonferroni inequality gives that

$$
\Sigma^{-}(u)-\Sigma \Sigma_{1}(u)-\Sigma \Sigma_{2}(u) \leq \mathbb{P}\left\{\sup _{(s, t) \in E_{u, 1}} Z_{u}(\Delta(u) s, T-\Delta(u) t)>m(u)\right\} \leq \Sigma^{+}(u),
$$

where

$$
\Sigma^{ \pm}(u)=\sum_{k, l=0}^{N(u) \pm 1} \mathbb{P}\left\{\sup _{(s, t) \in I_{k, l}} Z_{u}(\Delta(u) s, T-\Delta(u) t)>m(u)\right\}
$$

and for $i=1,2$

$$
\Sigma \Sigma_{i}(u)=\sum_{\left(k, l, k^{\prime}, l^{\prime}\right) \in \Lambda_{i}} \mathbb{P}\left\{\sup _{(s, t) \in I_{k, l}} Z_{u}(\Delta(u) s, T-\Delta(u) t)>m(u), \sup _{(s, t) \in I_{k^{\prime}, l^{\prime}}} Z_{u}(\Delta(u) s, T-\Delta(u) t)>m(u)\right\} .
$$

Upper or Lower bounds for $\Sigma^{ \pm}(u)$. By Lemma 3.1, we have

$$
\lim _{u \rightarrow \infty} \sup _{(s, t) \neq(0,0),(s, t) \in E_{u, 1}}\left|\frac{1-\sqrt{\operatorname{Var}\left(Z_{u}(\Delta(u) s, T-\Delta(u) t)\right)}}{\Delta(u) \frac{H}{T} t+\Delta(u) \frac{H}{T} s+\frac{(\Delta(u))^{2 H}}{2 u} s^{2 H}}-1\right|=0 .
$$

Thus for any $0<\epsilon<1$, let

$$
m_{k, l}^{ \pm \epsilon}(u)=m(u)\left(1+(1 \pm \epsilon)\left(\Delta(u) \frac{H}{T}(l \pm 1) S+\Delta(u) \frac{H}{T}(k \pm 1) S+\frac{(\Delta(u))^{2 H}}{2 u}(k \pm 1)^{2 H} S^{2 H}\right)\right)
$$

Moreover, denote by

$$
Z_{u, k, l}(s, t)=\frac{Z_{u}(\Delta(u)(k S+s), T-\Delta(u)(l S+t))}{\sqrt{\operatorname{Var}\left(Z_{u}(\Delta(u)(k S+s), T-\Delta(u)(l S+t))\right)}} .
$$

Then we have

$$
\begin{aligned}
& \Sigma^{+}(u) \leq \sum_{k, l=0}^{N(u)+1} \mathbb{P}\left\{\sup _{(s, t) \in[0, S]^{2}} Z_{u, k, l}(s, t)>m_{k, l}^{-\epsilon}(u)\right\} \\
& \Sigma^{-}(u) \geq \sum_{k, l=0}^{N(u)-1} \mathbb{P}\left\{\sup _{(s, t) \in[0, S]^{2}} Z_{u, k, l}(s, t)>m_{k, l}^{+\epsilon}(u)\right\} .
\end{aligned}
$$

Note that (7) implies that

$$
\lim _{u \rightarrow \infty} \sup _{(s, t) \neq\left(s^{\prime}, t^{\prime}\right),(s, t),\left(s^{\prime}, t^{\prime}\right) \in[0, S]^{2}}\left|\left(m_{k, l}^{ \pm \epsilon}(u)\right)^{2} \frac{1-\operatorname{Corr}\left(Z_{u, k, l}(s, t), Z_{u, k, l}\left(s^{\prime}, t^{\prime}\right)\right)}{\left|s-s^{\prime}\right|^{2 H}+\left|t-t^{\prime}\right|^{2 H}}-1\right|=0 .
$$

Thus by Lemma 4.2 , we have that

$$
\lim _{u \rightarrow \infty} \sup _{0 \leq k, l \leq N(u)+1}\left|\frac{\mathbb{P}\left\{\sup _{(s, t) \in[0, S]^{2}} Z_{u, k, l}(s, t)>m_{k, l}^{ \pm \epsilon}(u)\right\}}{\Psi\left(m_{k, l}^{ \pm \epsilon}(u)\right)}-\left(\mathcal{H}_{H}([0, S])\right)^{2}\right|=0
$$

This implies that

$$
\Sigma^{+}(u) \leq\left(\mathcal{H}_{H}([0, S])\right)^{2} \sum_{k, l=0}^{N(u)+1} \Psi\left(m_{k, l}^{-\epsilon}(u)\right)=\left(\frac{\mathcal{H}_{H}([0, S])}{S}\right)^{2} \Psi(m(u)) \Theta^{-}(u, S, \epsilon),
$$

and

$$
\Sigma^{-}(u) \geq\left(\mathcal{H}_{H}([0, S])\right)^{2} \sum_{k, l=0}^{N(u)-1} \Psi\left(m_{k, l}^{+\epsilon}(u)\right)=\left(\frac{\mathcal{H}_{H}([0, S])}{S}\right)^{2} \Psi(m(u)) \Theta^{+}(u, S, \epsilon),
$$

where

$\Theta^{ \pm}(u, S, \epsilon)=S^{2} \sum_{k, l=0}^{N(u) \mp 1} \exp \left(-(1 \pm \epsilon) m^{2}(u)\left(\Delta(u) \frac{H}{T}(l \pm 1) S+\Delta(u) \frac{H}{T}(k \pm 1) S+\frac{(\Delta(u))^{2 H}}{2 u}(k \pm 1)^{2 H} S^{2 H}\right)\right)$. 
Next we analyze $\Theta^{ \pm}(u, S, \epsilon)$. Note that for $\frac{1}{4}<H<\frac{1}{2}$

$$
\sup _{0 \leq k \leq N(u)+1} m^{2}(u) \frac{(\Delta(u))^{2 H}}{2 u}|k-1|^{2 H} S^{2 H} \leq Q(m(u))^{2-4 H} \frac{(\ln m(u))^{4 H}}{u} \leq Q u^{1-4 H}(\ln u)^{4 H} \rightarrow 0
$$

Hence, setting

$$
v(u, \epsilon)=(1-\epsilon) m^{2}(u) \Delta(u) \frac{H}{T}
$$

it follows that

$$
\begin{aligned}
\Theta^{-}(u, S, \epsilon) & \leq S^{2} \sum_{k, l=0}^{N(u)+1} e^{-(v(u, \epsilon)(l-1) S+v(u, \epsilon)(k-1) S)} \\
& =(v(u, \epsilon))^{-2}\left(\sum_{l=0}^{N(u)+1} e^{-v(u, \epsilon)(l-1) S} v(u, \epsilon) S\right)\left(\sum_{k=0}^{N(u)+1} e^{-v(u, \epsilon)(k-1) S} v(u, \epsilon) S\right) \\
& \leq(v(u, \epsilon))^{-2}\left(\int_{0}^{\infty} e^{-t} d t\right)^{2} \\
& \sim\left(m^{2}(u) \Delta(u)\right)^{-2}\left(\frac{T}{H}\right)^{2}=\left(H^{-1} 2^{-\frac{1}{2 H}} T^{2 H-1}\right)^{2} u^{\frac{2}{H}-4}, \quad u \rightarrow \infty, \epsilon \rightarrow 0,
\end{aligned}
$$

which together with the fact that

$$
\lim _{S \rightarrow \infty} \frac{\mathcal{H}_{H}([0, S])}{S}=\mathcal{H}_{H}
$$

leads to

$$
\Sigma^{+}(u) \leq\left(H^{-1} 2^{-\frac{1}{2 H}} T^{2 H-1} \mathcal{H}_{H}\right)^{2} u^{\frac{2}{H}-4} \Psi(m(u)), \quad u \rightarrow \infty
$$

Similarly, we can show that

$$
\Theta^{+}(u, S, \epsilon) \geq\left(H^{-1} 2^{-\frac{1}{2 H}} T^{2 H-1}\right)^{2} u^{\frac{2}{H}-4}, \quad u \rightarrow \infty, \epsilon \rightarrow 0, S \rightarrow \infty .
$$

Hence

$$
\Sigma^{-}(u) \geq\left(H^{-1} 2^{-\frac{1}{2 H}} T^{2 H-1} \mathcal{H}_{H}\right)^{2} u^{\frac{2}{H}-4} \Psi(m(u)), \quad u \rightarrow \infty
$$

Upper bounds of $\Sigma \Sigma_{i}(u), i=1,2$. For $\left(k, l, k^{\prime}, l^{\prime}\right) \in \Lambda_{1}$, without loss of generality, we assume that $k^{\prime}=k+1$. We next split $I_{k^{\prime}, l^{\prime}}$ into two parts: the first part has smaller volume than the original one and the second part is separated by a positive distance away from $I_{k, l}$. Then we can show that the double-sum term with index in $\Lambda_{1}(u)$ is negligible due to the smaller volume of the first one and the positive distance of the second one from $I_{k, l}$. Denote by

$$
I_{k^{\prime}, l^{\prime}}^{(1)}=[(k+1) S,(k+1) S+\sqrt{S}] \times\left[l^{\prime} S,\left(l^{\prime}+1\right) S\right], \quad I_{k^{\prime}, l^{\prime}}^{(2)}=[(k+1) S+\sqrt{S},(k+2) S,] \times\left[l^{\prime} S,\left(l^{\prime}+1\right) S\right] .
$$

Hence, for $\left(k, l, k^{\prime}, l^{\prime}\right) \in \Lambda_{1}$,

$$
\begin{aligned}
& \mathbb{P}\left\{\sup _{(s, t) \in I_{k, l}} Z_{u}(\Delta(u) s, T-\Delta(u) t)>m(u), \sup _{(s, t) \in I_{k^{\prime}, l^{\prime}}} Z_{u}(\Delta(u) s, T-\Delta(u) t)>m(u)\right\} \\
& \leq \mathbb{P}\left\{\sup _{(s, t) \in I_{k, l}} \bar{Z}_{u}(\Delta(u) s, T-\Delta(u) t)>m_{k, l}^{-\epsilon}(u), \sup _{(s, t) \in I_{k^{\prime}, l^{\prime}}^{(2)}} \bar{Z}_{u}(\Delta(u) s, T-\Delta(u) t)>m_{k^{\prime}, l^{\prime}}^{-\epsilon}(u)\right\} \\
& +\mathbb{P}\left\{\sup _{(s, t) \in I_{k^{\prime}, l^{\prime}}^{(1)}} \bar{Z}_{u}(\Delta(u) s, T-\Delta(u) t)>m_{k^{\prime}, l^{\prime}}^{-\epsilon}(u)\right\},
\end{aligned}
$$

where

$$
\bar{Z}_{u}(\Delta(u) s, T-\Delta(u) t)=\frac{Z_{u}(\Delta(u) s, T-\Delta(u) t)}{\sqrt{\operatorname{Var}\left(Z_{u}(\Delta(u) s, T-\Delta(u) t)\right.}}
$$


Noting that (10) holds and

$$
\mathbb{P}\left\{\sup _{(s, t) \in I_{k^{\prime}, l^{\prime}}^{(1)}} \bar{Z}_{u}(\Delta(u) s, T-\Delta(u) t)>m_{k^{\prime}, l^{\prime}}^{-\epsilon}(u)\right\}=\mathbb{P}\left\{\sup _{(s, t) \in[0, \sqrt{S}] \times[0, S]} Z_{u, k^{\prime}, l^{\prime}}(s, t)>m_{k^{\prime}, l^{\prime}}^{-\epsilon}(u)\right\},
$$

by Lemma 4.2 in Appendix, we have that

$$
\lim _{u \rightarrow \infty} \sup _{0 \leq k^{\prime}, l^{\prime} \leq N(u)+1}\left|\frac{\mathbb{P}\left\{\sup _{(s, t) \in[0, \sqrt{S}] \times[0, S]} Z_{u, k^{\prime}, l^{\prime}}(s, t)>m_{k^{\prime}, l^{\prime}}^{ \pm \epsilon}(u)\right\}}{\Psi\left(m_{k^{\prime}, l^{\prime}}^{-\epsilon}(u)\right)}-\mathcal{H}_{H}([0, \sqrt{S}]) \mathcal{H}_{H}([0, S])\right|=0 .
$$

Using also the fact that $I_{k, l}$ has at most 8 neighborhoods and

$$
\lim _{S \rightarrow \infty} \frac{\mathcal{H}_{H}([0, \sqrt{S}])}{S}=\lim _{S \rightarrow \infty} \frac{\mathcal{H}_{H}([0, \sqrt{S}])}{\sqrt{S}} \lim _{S \rightarrow \infty} S^{-\frac{1}{2}}=\mathcal{H}_{H} \lim _{S \rightarrow \infty} S^{-\frac{1}{2}}=0
$$

in light of (11) and (15), we have

$$
\begin{aligned}
& \sum_{\left(k, l, k^{\prime}, l^{\prime}\right) \in \Lambda_{1}} \mathbb{P}\left\{\sup _{(s, t) \in I_{k^{\prime}, l^{\prime}}^{(1)}} \bar{Z}_{u}(\Delta(u) s, T-\Delta(u) t)>m_{k^{\prime}, l^{\prime}}^{-\epsilon}(u)\right\} \\
& \leq 8 \sum_{k^{\prime}, l^{\prime}=0}^{N(u)+1} \mathcal{H}_{H}([0, \sqrt{S}]) \mathcal{H}_{H}([0, S]) \Psi\left(m_{k^{\prime}, l^{\prime}}^{-\epsilon}(u)\right) \\
& \leq 8 \frac{\mathcal{H}([0, \sqrt{S}])}{S} \frac{\mathcal{H}([0, S])}{S}\left(H^{-1} 2^{-\frac{1}{2 H}} T^{2 H-1}\right)^{2} u^{\frac{2}{H}-4} \Psi(m(u)) \\
& =o\left(u^{\frac{2}{H}-4} \Psi(m(u))\right), \quad u \rightarrow \infty, S \rightarrow \infty .
\end{aligned}
$$

Lemma 3.3 shows that for $u$ sufficiently large and $(s, t),\left(s^{\prime}, t^{\prime}\right) \in E_{u, 1}$

$$
\operatorname{Corr}\left(\bar{Z}_{u}(\Delta(u) s, T-\Delta(u) t), \bar{Z}_{u}\left(\Delta(u) s^{\prime}, T-\Delta(u) t^{\prime}\right)\right)>0
$$

and

$$
\lim _{u \rightarrow \infty} \sup _{(s, t) \neq\left(s^{\prime}, t^{\prime}\right),(s, t),\left(s^{\prime}, t^{\prime}\right) \in E_{u, 1}}\left|(m(u))^{2} \frac{1-\operatorname{Corr}\left(\bar{Z}_{u}(\Delta(u) s, T-\Delta(u) t), \bar{Z}_{u}\left(\Delta(u) s^{\prime}, T-\Delta(u) t^{\prime}\right)\right)}{\left|s-s^{\prime}\right|^{2 H}+\left|t-t^{\prime}\right|^{2 H}}-1\right|=0 .
$$

Hence by Lemma 4.3 in Appendix, there exist constants $\mathcal{C}, \mathcal{C}_{1}>0$ such that for $\left(k, l, k^{\prime}, l^{\prime}\right) \in \Lambda_{1}$ and $u$ sufficiently large

$$
\begin{aligned}
& \mathbb{P}\left\{\sup _{(s, t) \in I_{k, l}} \bar{Z}_{u}(\Delta(u) s, T-\Delta(u) t)>m_{k, l}^{-\epsilon}(u), \sup _{(s, t) \in I_{k^{\prime}, l^{\prime}}^{(2)}} \bar{Z}_{u}(\Delta(u) s, T-\Delta(u) t)>m_{k^{\prime}, l^{\prime}}^{-\epsilon}(u)\right\} \\
& \leq \mathcal{C} S^{4} e^{-\mathcal{C}_{1} S^{\frac{H}{2}}} \Psi\left(m_{k, l, k^{\prime}, l^{\prime}}^{-\epsilon}(u)\right) ;
\end{aligned}
$$

and for $\left(k, l, k^{\prime}, l^{\prime}\right) \in \Lambda_{2}$ and $u$ sufficiently large

$$
\begin{aligned}
& \mathbb{P}\left\{\sup _{(s, t) \in I_{k, l}} \bar{Z}_{u}(\Delta(u) s, T-\Delta(u) t)>m_{k, l}^{-\epsilon}(u), \sup _{(s, t) \in I_{k^{\prime}, l^{\prime}}} \bar{Z}_{u}(\Delta(u) s, T-\Delta(u) t)>m_{k^{\prime}, l^{\prime}}^{-\epsilon}(u)\right\} \\
& \leq \mathcal{C} S^{4} e^{-\mathcal{C}_{1}\left(\left|k-k^{\prime}\right|^{2}+\left|l-l^{\prime}\right|^{2}\right)^{\frac{H}{2}} S^{H}} \Psi\left(m_{k, l, k^{\prime}, l^{\prime}}^{-\epsilon}(u)\right),
\end{aligned}
$$

where

$$
m_{k, l, k^{\prime}, l^{\prime}}^{-\epsilon}(u)=\min \left(m_{k, l}^{-\epsilon}(u), m_{k^{\prime}, l^{\prime}}^{-\epsilon}(u)\right) .
$$

Consequently, noting that $I_{k, l}$ has at most 8 neighborhoods and in light of (11) and (15)

$$
\sum_{\left(k, l, k^{\prime}, l^{\prime}\right) \in \Lambda_{1}} \mathbb{P}\left\{\sup _{(s, t) \in I_{k, l}} \bar{Z}_{u}(\Delta(u) s, T-\Delta(u) t)>m_{k, l}^{-\epsilon}(u), \sup _{(s, t) \in I_{k^{\prime}, l^{\prime}}^{(2)}} \bar{Z}_{u}(\Delta(u) s, T-\Delta(u) t)>m_{k^{\prime}, l^{\prime}}^{-\epsilon}(u)\right\}
$$




$$
\begin{aligned}
& \leq \sum_{\left(k, l, k^{\prime}, l^{\prime}\right) \in \Lambda_{1}} \mathcal{C} S^{4} e^{-\mathcal{C}_{1} S^{\frac{H}{2}}} \Psi\left(m_{k, l, k^{\prime}, l^{\prime}}^{-\epsilon}(u)\right) \\
& \leq \sum_{\left(k, l, k^{\prime}, l^{\prime}\right) \in \Lambda_{1}} \mathcal{C} S^{4} e^{-\mathcal{C}_{1} S^{\frac{H}{2}}}\left(\Psi\left(m_{k, l}^{-\epsilon}(u)\right)+\Psi\left(m_{k^{\prime}, l^{\prime}}^{-\epsilon}(u)\right)\right) \\
& \leq \sum_{k, l=0}^{N(u)+1} 16 \mathcal{C} S^{4} e^{-\mathcal{C}_{1} S^{\frac{H}{2}}} \Psi\left(m_{k, l}^{-\epsilon}(u)\right) \\
& \leq Q S^{2} e^{-\mathcal{C}_{1} S^{\frac{H}{2}}} u^{\frac{2}{H}-4} \Psi(m(u))=o\left(u^{\frac{2}{H}-4} \Psi(m(u))\right), \quad u \rightarrow \infty, S \rightarrow \infty .
\end{aligned}
$$

Therefore, we can conclude that

$$
\Sigma \Sigma_{1}(u)=o\left(u^{\frac{2}{H}-4} \Psi(m(u))\right), \quad u \rightarrow \infty, S \rightarrow \infty .
$$

Moreover, by (19) and (11)-(15)

$$
\begin{aligned}
\Sigma \Sigma_{2}(u) & \leq \sum_{\left(k, l, k^{\prime}, l^{\prime}\right) \in \Lambda_{2}} \mathbb{P}\left\{\sup _{(s, t) \in I_{k, l}} \bar{Z}_{u}(\Delta(u) s, T-\Delta(u) t)>m_{k, l}^{-\epsilon}(u), \sup _{(s, t) \in I_{k^{\prime}, l^{\prime}}} \bar{Z}_{u}(\Delta(u) s, T-\Delta(u) t)>m_{k^{\prime}, l^{\prime}}^{-\epsilon}(u)\right\} \\
& \leq \sum_{\left(k, l, k^{\prime}, l^{\prime}\right) \in \Lambda_{2}} \mathcal{C} S^{4} e^{-\mathcal{C}_{1}\left(\left|k-k^{\prime}\right|^{2}+\left|l-l^{\prime}\right|^{2}\right)^{\frac{H}{2}} S^{H}} \Psi\left(m_{k, l, k^{\prime}, l^{\prime}}^{-\epsilon}(u)\right) \\
& \leq \sum_{k, l=0}^{N(u)+1} \Psi\left(m_{k, l}^{-\epsilon}(u)\right) 2 \mathcal{C} S^{4} \sum_{k^{\prime}, l^{\prime} \geq 0, k^{\prime}+l^{\prime} \neq 0} e^{-\mathcal{C}_{1}\left(k^{\prime 2}+l^{\prime 2}\right)^{\frac{H}{2}} S^{H}} \\
& \leq \sum_{k, l=0}^{N(u)+1} Q S^{4} e^{-Q_{1} S^{H}} \Psi\left(m_{k, l}^{-\epsilon}(u)\right) \\
(21) \quad \leq & Q S^{2} e^{-Q_{1} S^{H}} u^{\frac{2}{H}-4} \Psi(m(u))=o\left(u^{\frac{2}{H}-4} \Psi(m(u))\right), \quad u \rightarrow \infty, S \rightarrow \infty .
\end{aligned}
$$

Inserting (16)-(17) and (20)-(21) into (8), we deduce that

$$
\mathbb{P}\left\{\sup _{(s, t) \in E_{u, 1}} Z_{u}(\Delta(u) s, T-\Delta(u) t)>m(u)\right\} \sim\left(H^{-1} 2^{-\frac{1}{2 H}} T^{2 H-1} \mathcal{H}_{H}\right)^{2} u^{\frac{2}{H}-4} \Psi(m(u)), \quad u \rightarrow \infty,
$$

which together with (4) and (5) establishes the claim.

Case $H=\frac{1}{4}$. Note that (8)-(12) still hold for $H=\frac{1}{4}$. We proceed along the same line of reasoning in the proof of the case $\frac{1}{4}<H<\frac{1}{2}$. We begin with the analysis of $\Theta^{ \pm}(u, S, \epsilon)$. In contrast to the case $\frac{1}{4}<H<\frac{1}{2}$, it will be shown that

$$
m^{2}(u) \frac{(\Delta(u))^{2 H}}{2 u}(k-1)^{2 H} S^{2 H}
$$

is not negligible and also contributes to the asymptotics of $\Theta^{ \pm}(u, S, \epsilon)$. Recalling that

$$
v(u, \epsilon)=(1-\epsilon) m^{2}(u) \Delta(u) \frac{H}{T},
$$

it follows that

$$
\begin{aligned}
\Theta^{-}(u, S, \epsilon) & =S^{2} \sum_{k, l=0}^{N(u)+1} \exp \left(-\left(v(u, \epsilon)(l-1) S+v(u, \epsilon)(k-1) S+m^{2}(u) \frac{(\Delta(u))^{2 H}}{2 u}(k-1)^{2 H} S^{2 H}\right)\right) \\
& =\sum_{l=0}^{N(u)+1} e^{-v(u, \epsilon)(l-1) S} S \sum_{k=0}^{N(u)+1} \exp \left(-\left(v(u, \epsilon)(k-1) S+(1-\epsilon) m^{2}(u) \frac{(\Delta(u))^{2 H}}{2 u}(k-1)^{2 H} S^{2 H}\right)\right) S
\end{aligned}
$$

The first sum satisfies

$$
\sum_{l=0}^{N(u)+1} e^{-v(u, \epsilon)(l-1) S} S=(v(u, \epsilon))^{-1} \sum_{l=0}^{N(u)+1} e^{-v(u, \epsilon)(l-1) S} v(u, \epsilon) S
$$




$$
\leq(v(u, \epsilon))^{-1} \int_{0}^{\infty} e^{-t} d t \sim\left(m^{2}(u) \Delta(u) \frac{H}{T}\right)^{-1}, \quad u \rightarrow \infty, \epsilon \rightarrow 0 .
$$

For the second one

$$
\begin{aligned}
& \sum_{k=0}^{N(u)+1} \exp \left(-\left(v(u, \epsilon)(k-1) S+(1-\epsilon) m^{2}(u) \frac{(\Delta(u))^{2 H}}{2 u}(k-1)^{2 H} S^{2 H}\right)\right) S \\
= & (v(u, \epsilon))^{-1} \sum_{k=0}^{N(u)+1} \exp \left(-v(u, \epsilon)(k-1) S+\left(\frac{(1-\epsilon)^{\frac{1}{2 H}} 2^{-\frac{1}{2 H}} u^{-\frac{1}{2 H}}(m(u))^{\frac{1}{H}} \Delta(u)}{v(u, \epsilon)} v(u, \epsilon)(k-1) S\right)^{2 H}\right) v(u, \epsilon) S .
\end{aligned}
$$

Note that for $H=\frac{1}{4}$,

$$
\frac{(1-\epsilon)^{\frac{1}{2 H}} 2^{-\frac{1}{2 H}} u^{-\frac{1}{2 H}}(m(u))^{\frac{1}{H}} \Delta(u)}{v(u, \epsilon)} \sim \sqrt{T}(1-\epsilon), \quad u \rightarrow \infty .
$$

Thus

$$
\begin{aligned}
& \sum_{k=0}^{N(u)+1} \exp \left(-\left(v(u, \epsilon)(k-1) S+(1-\epsilon) m^{2}(u) \frac{(\Delta(u))^{2 H}}{2 u}(k-1)^{2 H} S^{2 H}\right)\right) S \\
& \sim\left(m^{2}(u) \Delta(u) \frac{H}{T}\right)^{-1} \int_{0}^{\infty} e^{-x-T^{\frac{1}{4}} \sqrt{x}} d x, \quad u \rightarrow \infty, \epsilon \rightarrow 0 .
\end{aligned}
$$

Consequently,

$$
\Theta^{-}(u, S, \epsilon) \leq\left(m^{2}(u) \Delta(u) \frac{H}{T}\right)^{-2} \int_{0}^{\infty} e^{-x-T^{\frac{1}{4}} \sqrt{x}} d x, \quad u \rightarrow \infty, \epsilon \rightarrow 0 .
$$

Similarly,

$$
\Theta^{+}(u, S, \epsilon) \geq\left(m^{2}(u) \Delta(u) \frac{H}{T}\right)^{-2} \int_{0}^{\infty} e^{-x-T^{\frac{1}{4}} \sqrt{x}} d x, \quad u \rightarrow \infty, \epsilon \rightarrow 0 .
$$

In light of (11) and (12), we have that

$$
\begin{aligned}
\Sigma^{-}(u) & \leq\left(\frac{\mathcal{H}_{H}([0, S])}{S}\right)^{2}\left(m^{2}(u) \Delta(u) \frac{H}{T}\right)^{-2} \int_{0}^{\infty} e^{-x-T^{\frac{1}{4}} \sqrt{x}} d x \Psi(m(u)) \\
& \leq\left(H^{-1} 2^{-\frac{1}{2 H}} T^{2 H-1} \mathcal{H}_{H}\right)^{2} \int_{0}^{\infty} e^{-x-T^{\frac{1}{4}} \sqrt{x}} d x u^{\frac{2}{H}-4} \Psi(m(u)), \quad u \rightarrow \infty, S \rightarrow \infty, \\
\Sigma^{+}(u) & \geq\left(H^{-1} 2^{-\frac{1}{2 H}} T^{2 H-1} \mathcal{H}_{H}\right)^{2} \int_{0}^{\infty} e^{-x-T^{\frac{1}{4}} \sqrt{x}} d x u^{\frac{2}{H}-4} \Psi(m(u)), \quad u \rightarrow \infty, S \rightarrow \infty .
\end{aligned}
$$

The negligibility of $\Sigma \Sigma_{i}(u), i=1,2$ holds due to the fact that (18)-(21) are also valid for $H=\frac{1}{4}$. Therefore we have, as $u \rightarrow \infty$,

$$
\mathbb{P}\left\{\sup _{(s, t) \in E_{u, 1}} Z_{u}(\Delta(u) s, T-\Delta(u) t)>m(u)\right\} \sim\left(H^{-1} 2^{-\frac{1}{2 H}} T^{2 H-1} \mathcal{H}_{H}\right)^{2} \int_{0}^{\infty} e^{-x-T^{\frac{1}{4}} \sqrt{x}} d x u^{\frac{2}{H}-4} \Psi(m(u)),
$$

which combined with (4) and (5) establishes the claim.

Case $0<H<\frac{1}{4}$. For $0<H<\frac{1}{4}$, (8)-(12) are still satisfied. Thus we proceed as in the proof of $\frac{1}{4}<H<\frac{1}{2}$. We next analyze $\Theta^{ \pm}(u, S, \epsilon)$. As it will be shown below, term

$$
m^{2}(u) \frac{(\Delta(u))^{2 H}}{2 u}(k-1)^{2 H} S^{2 H}
$$

plays a crucial role to the asymptotics of $\Theta^{ \pm}(u, S, \epsilon)$. Denote by

$$
v^{\prime}(u, \epsilon)=(1-\epsilon)^{\frac{1}{2 H}} 2^{-\frac{1}{2 H}} u^{-\frac{1}{2 H}}(m(u))^{\frac{1}{H}} \Delta(u),
$$

it follows that

$$
\Theta^{-}(u, S, \epsilon)=S^{2} \sum_{k, l=0}^{N(u)+1} \exp \left(-(1-\epsilon) m^{2}(u)\left(\Delta(u) \frac{H}{T}(l-1) S+\Delta(u) \frac{H}{T}(k-1) S+\frac{(\Delta(u))^{2 H}}{2 u}(k-1)^{2 H} S^{2 H}\right)\right)
$$




$$
=\sum_{l=0}^{N(u)+1} e^{-v(u, \epsilon)(l-1) S} S \sum_{k=0}^{N(u)+1} \exp \left(-\left(v(u, \epsilon)(k-1) S+\left(v^{\prime}(u, \epsilon)(k-1) S\right)^{2 H}\right)\right) S,
$$

where $v(u, \epsilon)$ is defined in (14). The first sum satisfies (22) with $0<H<1 / 4$. For the second sum

$$
\begin{aligned}
& \sum_{k=0}^{N(u)+1} \exp \left(-\left(v(u, \epsilon)(k-1) S+\left(v^{\prime}(u, \epsilon)(k-1) S\right)^{2 H}\right)\right) S \\
= & \left(v^{\prime}(u, \epsilon)\right)^{-1} \sum_{k=0}^{N(u)+1} \exp \left(-\gamma(u) v^{\prime}(u, \epsilon)(k-1) S-\left(v^{\prime}(u, \epsilon)(k-1) S\right)^{2 H}\right) v^{\prime}(u, \epsilon) S,
\end{aligned}
$$

where

$$
\gamma(u)=\frac{v(u, \epsilon)}{v^{\prime}(u, \epsilon)}=\frac{(1-\epsilon) m^{2}(u) \Delta(u) \frac{H}{T}}{(1-\epsilon)^{\frac{1}{2 H}} 2^{-\frac{1}{2 H}} u^{-\frac{1}{2 H}}(m(u))^{\frac{1}{H}} \Delta(u)} \sim Q u^{2-\frac{1}{2 H}} \rightarrow 0, \quad u \rightarrow \infty .
$$

Thus

$$
\begin{aligned}
\sum_{k=0}^{N(u)+1} \exp \left(-\left(v(u, \epsilon)(k-1) S+\left(v^{\prime}(u, \epsilon)(k-1) S\right)^{2 H}\right)\right) S & \sim\left(v^{\prime}(u, \epsilon)\right)^{-1} \int_{0}^{\infty} e^{-x^{2 H}} d x \\
& \sim \Gamma\left(\frac{1}{2 H}+1\right) T^{-1} u^{\frac{1}{2 H}}, \quad u \rightarrow \infty, \epsilon \rightarrow 0 .
\end{aligned}
$$

Consequently,

$$
\Theta^{-}(u, S, \epsilon) \leq H^{-1} 2^{-\frac{1}{2 H}} T^{2 H-2} \Gamma\left(\frac{1}{2 H}+1\right) u^{\frac{3}{2 H}-2}, \quad u \rightarrow \infty, \epsilon \rightarrow 0
$$

Similarly,

$$
\Theta^{+}(u, S, \epsilon) \geq H^{-1} 2^{-\frac{1}{2 H}} T^{2 H-2} \Gamma\left(\frac{1}{2 H}+1\right) u^{\frac{3}{2 H}-2}, \quad u \rightarrow \infty, \epsilon \rightarrow 0 .
$$

In light of (11) and (12), we have that, as $u \rightarrow \infty, S \rightarrow \infty$,

$$
\begin{aligned}
& \Sigma^{-}(u) \leq H^{-1} 2^{-\frac{1}{2 H}} T^{2 H-2} \Gamma\left(\frac{1}{2 H}+1\right)\left(\mathcal{H}_{H}\right)^{2} u^{\frac{3}{2 H}-2} \Psi(m(u)), \\
& \Sigma^{+}(u) \geq H^{-1} 2^{-\frac{1}{2 H}} T^{2 H-2} \Gamma\left(\frac{1}{2 H}+1\right)\left(\mathcal{H}_{H}\right)^{2} u^{\frac{3}{2 H}-2} \Psi(m(u)) .
\end{aligned}
$$

Following line by line the same as in (18)-(21), we can show that, for $i=1,2$,

$$
\Sigma \Sigma_{i}(u)=o\left(u^{\frac{3}{2 H}-2} \Psi(m(u))\right), \quad u \rightarrow \infty, S \rightarrow \infty .
$$

Therefore, we conclude that, as $u \rightarrow \infty$,

$$
\mathbb{P}\left\{\sup _{(s, t) \in E_{u, 1}} Z_{u}(\Delta(u) s, T-\Delta(u) t)>m(u)\right\} \sim H^{-1} 2^{-\frac{1}{2 H}} T^{2 H-2} \Gamma\left(\frac{1}{2 H}+1\right)\left(\mathcal{H}_{H}\right)^{2} u^{\frac{3}{2 H}-2} \Psi(m(u)),
$$

which establishes the claim with the aid of (4) and (5). This completes the proof.

Proof of Theorem 2.2: We distinguish between $H \geq \frac{1}{2}$ and $H<\frac{1}{2}$.

Case $H \geq \frac{1}{2}$. We have that

$$
\begin{aligned}
\mathbb{P}\left\{\sup _{0 \leq t \leq T} U_{t}>u\right\} & =\mathbb{P}\left\{\sup _{(s, t) \in A}\left(X_{t}-X_{s}\right)>u\right\} \\
& =\mathbb{P}\left\{\sup _{(s, t) \in A}\left(B_{H}(t)-B_{H}(s)-\frac{1}{2}\left(t^{2 H}-s^{2 H}\right)+\mu(t-s)\right)>u\right\} \\
& =\mathbb{P}\left\{\sup _{(s, t) \in A} Z_{u, 1}(s, t)>m_{1}(u)\right\}
\end{aligned}
$$


where

$$
Z_{u, 1}(s, t)=\frac{B_{H}(t)-B_{H}(s)}{u-\mu(t-s)+\frac{1}{2}\left(t^{2 H}-s^{2 H}\right)} m_{1}(u), \quad m_{1}(u)=\frac{u-\mu T+\frac{1}{2} T^{2 H}}{T^{H}} .
$$

Furthermore,

$$
\begin{aligned}
\mathbb{P}\left\{\sup _{(s, t) \in E_{u, 2}} Z_{u, 1}(s, t)>m_{1}(u)\right\} & \leq \mathbb{P}\left\{\sup _{0 \leq t \leq T} U_{t}>u\right\} \\
& \leq \mathbb{P}\left\{\sup _{(s, t) \in E_{u, 2}} Z_{u, 1}(s, t)>m_{1}(u)\right\}+\mathbb{P}\left\{\sup _{(s, t) \in A \backslash E_{u, 2}} Z_{u, 1}(s, t)>m_{1}(u)\right\},
\end{aligned}
$$

where

$$
E_{u, 2}=\left[0,\left(\ln m_{1}(u)\right)^{2} /\left(m_{1}(u)\right)^{2}\right] \times\left[T-\left(\ln m_{1}(u)\right)^{2} /\left(m_{1}(u)\right)^{2}, T\right] .
$$

In light of Lemma 3.2, it follows that, for $u$ sufficiently large,

$$
\sup _{(s, t) \in A \backslash E_{u, 2}} \sqrt{\operatorname{Var}\left(Z_{u, 1}(s, t)\right)} \leq 1-Q\left(\frac{\ln m_{1}(u)}{m_{1}(u)}\right)^{2} .
$$

Moreover, direct calculation shows that

$$
\mathbb{E}\left(\left(Z_{u, 1}(s, t)-Z_{u, 1}\left(s^{\prime}, t^{\prime}\right)\right)^{2}\right) \leq Q_{1}\left(\left|t-t^{\prime}\right|^{2 H}+\left|s-s^{\prime}\right|^{2 H}\right), \quad(s, t),\left(s^{\prime}, t^{\prime}\right) \in A .
$$

Using Piterbarg Theorem (Theorem 8.1 in [18]), we have, for $u$ sufficiently large,

$$
\mathbb{P}\left\{\sup _{(s, t) \in A \backslash E_{u, 2}} Z_{u, 1}(s, t)>m_{1}(u)\right\} \leq Q_{2}\left(m_{1}(u)\right)^{\frac{2}{H}} \Psi\left(\frac{m_{1}(u)}{1-Q\left(\frac{\ln m_{1}(u)}{m_{1}(u)}\right)^{2}}\right) .
$$

Next we focus on $\mathbb{P}\left\{\sup _{(s, t) \in E_{u, 2}} Z_{u, 1}(s, t)>m_{1}(u)\right\}$. Lemmas 3.1 and 3.3 lead to

$$
\begin{gathered}
\lim _{u \rightarrow \infty} \sup _{(s, t) \neq(0, T),(s, t) \in E_{u, 2}}\left|\frac{1-\sqrt{\operatorname{Var}\left(Z_{u, 1}(s, t)\right)}}{\frac{H(T-t)}{T}+\frac{H}{T} s}-1\right|=0, \\
\lim _{u \rightarrow \infty} \sup _{(s, t) \neq\left(s^{\prime}, t^{\prime}\right),(s, t),\left(s^{\prime}, t^{\prime}\right) \in E_{u, 2}}\left|\frac{1-\operatorname{Corr}\left(Z_{u, 1}(s, t), Z_{u, 1}\left(s^{\prime}, t^{\prime}\right)\right)}{\frac{\left|s-s^{\prime}\right|^{2 H}+\left|t-t^{\prime}\right|^{2 H}}{2 T^{2 H}}}-1\right|=0,
\end{gathered}
$$

which coincide with the local variance and correlation behavior of $Z_{u}(s, t)$ in proof of Theorem 2.1 for case $H \geq \frac{1}{2}$. Similarly as in proof of Theorem 2.1, we derive that, for $H>\frac{1}{2}$,

$$
\mathbb{P}\left\{\sup _{(s, t) \in E_{u, 2}} Z_{u, 1}(s, t)>m_{1}(u)\right\} \sim \Psi\left(m_{1}(u)\right), \quad u \rightarrow \infty
$$

and for $H=\frac{1}{2}$

$$
\mathbb{P}\left\{\sup _{(s, t) \in E_{u, 2}} Z_{u, 1}(s, t)>m_{1}(u)\right\} \sim\left(\mathcal{P}_{1 / 2}^{1}\right)^{2} \Psi\left(m_{1}(u)\right), \quad u \rightarrow \infty .
$$

Inserting the above asymptotics, (24) and (3) in (23), we establish the claim.

Case $0<H<\frac{1}{2}$. Observe that

$$
\begin{aligned}
\mathbb{P}\left\{\sup _{0 \leq t \leq T} U_{t}>u\right\} & =\mathbb{P}\left\{\sup _{(s, t) \in A}\left(X_{t}-X_{s}\right)>u\right\} \\
& =\mathbb{P}\left\{\sup _{(s, t) \in A}\left(B_{H}(t)-B_{H}(s)-\frac{1}{2}\left(t^{2 H}-s^{2 H}\right)+\mu(t-s)\right)>u\right\} \\
& =\mathbb{P}\left\{\sup _{(s, t) \in A} Z_{u, 2}(s, t)>m_{2}(u)\right\}
\end{aligned}
$$


where

$$
Z_{u, 2}(s, t)=\frac{B_{H}(t)-B_{H}(s)}{u-\mu(t-s)+\frac{1}{2}\left(t^{2 H}-s^{2 H}\right)} m_{2}(u), \quad m_{2}(u)=\inf _{0 \leq s \leq T} \frac{u-\mu(T-s)+\frac{1}{2}\left(T^{2 H}-s^{2 H}\right)}{(T-s)^{H}} .
$$

Thus we have

$$
\begin{aligned}
\mathbb{P}\left\{\sup _{(s, t) \in E_{u, 3}} Z_{u, 2}(s, t)>m_{2}(u)\right\} & \leq \mathbb{P}\left\{\sup _{0 \leq t \leq T} U_{t}>u\right\} \\
& \leq \mathbb{P}\left\{\sup _{(s, t) \in E_{u, 3}} Z_{u, 2}(s, t)>m_{2}(u)\right\}+\mathbb{P}\left\{\sup _{(s, t) \in A \backslash E_{u, 3}} Z_{u, 2}(s, t)>m_{2}(u)\right\},
\end{aligned}
$$

where

$$
E_{u, 3}=\left[0, s_{u}+\left(\ln m_{2}(u)\right) / m_{2}(u)\right] \times\left[T-\left(\ln m_{2}(u)\right)^{2} /\left(m_{2}(u)\right)^{2}, T\right] .
$$

In light of Lemma 3.2 , it follows that, for $u$ sufficiently large,

$$
\sup _{(s, t) \in A \backslash E_{u, 3}} \sqrt{\operatorname{Var}\left(Z_{u, 2}(s, t)\right)} \leq 1-Q_{3}\left(\frac{\ln m_{2}(u)}{m_{2}(u)}\right)^{2},
$$

and direct calculation shows that

$$
\mathbb{E}\left(\left(Z_{u, 2}(s, t)-Z_{u, 2}\left(s^{\prime}, t^{\prime}\right)\right)^{2}\right) \leq Q_{4}\left(\left|t-t^{\prime}\right|^{2 H}+\left|s-s^{\prime}\right|^{2 H}\right), \quad(s, t),\left(s^{\prime}, t^{\prime}\right) \in A .
$$

By Piterbarg Theorem, we have, for $u$ sufficiently large,

$$
\mathbb{P}\left\{\sup _{(s, t) \in A \backslash E_{u, 3}} Z_{u, 2}(s, t)>m_{2}(u)\right\} \leq Q_{5}\left(m_{2}(u)\right)^{\frac{2}{H}} \Psi\left(\frac{m_{2}(u)}{1-Q\left(\frac{\ln m_{2}(u)}{m_{2}(u)}\right)^{2}}\right) .
$$

Next we analyze the asymptotics of $\mathbb{P}\left\{\sup _{(s, t) \in E_{u, 3}} Z_{u, 2}(s, t)>m_{2}(u)\right\}$. Rewrite

$$
\mathbb{P}\left\{\sup _{(s, t) \in E_{u, 3}} Z_{u, 2}(s, t)>m_{2}(u)\right\}=\mathbb{P}\left\{\sup _{(s, t) \in E_{u, 4}} Z_{u, 2}\left(s_{u}+\Delta_{1}(u) s, T-\Delta_{1}(u) t\right)>m_{2}(u)\right\},
$$

where

$$
E_{u, 4}=\left[-s_{u} / \Delta_{1}(u),\left(\ln m_{2}(u)\right) /\left(m_{2}(u) \Delta_{1}(u)\right)\right] \times\left[0,\left(\ln m_{2}(u)\right)^{2} /\left(\left(m_{2}(u)\right)^{2} \Delta_{1}(u)\right)\right], \Delta_{1}(u)=2^{\frac{1}{2 H}} T\left(m_{2}(u)\right)^{-\frac{1}{H}},
$$

and $s_{u}$ is defined in Lemma 3.2. Lemmas 3.2 and 3.3 lead to

$$
\lim _{u \rightarrow \infty} \sup _{(s, t) \neq(0,0),(s, t) \in E_{u, 4}}\left|\frac{1-\sqrt{\operatorname{Var}\left(Z_{u, 2}\left(s_{u}+\Delta_{1}(u) s, T-\Delta_{1}(u) t\right)\right)}}{\frac{H(1-H)}{2 T^{2}}\left(\Delta_{1}(u)\right)^{2} s^{2}+\frac{H}{T} \Delta_{1}(u) t}-1\right|=0,
$$

and

$$
\lim _{u \rightarrow \infty} \sup _{(s, t) \neq\left(s^{\prime}, t^{\prime}\right),(s, t),\left(s^{\prime}, t^{\prime}\right) \in E_{u, 4}}\left|\frac{\left.\left.1-\operatorname{Corr}\left(Z_{u, 2}\left(s_{u}+\Delta_{1}(u) s, T-\Delta_{1}(u) t\right)\right), Z_{u, 2}\left(s_{u}+\Delta_{1}(u) s, T-\Delta_{1}(u) t\right)\right)\right)}{\left(m_{2}(u)\right)^{-2}\left(\left|s-s^{\prime}\right|^{2 H}+\left|t-t^{\prime}\right|^{2 H}\right)}-1\right|=0 .
$$

Next we check the conditions of Lemma 4.1 in Appendix. Following the same notation as in Lemma 4.1, we have that, for $0<H<\frac{1}{2}$,

$$
\begin{gathered}
\nu_{1}=\lim _{u \rightarrow \infty}\left(m_{2}(u)\right)^{2} \frac{H}{T} \Delta_{1}(u)=2^{\frac{1}{2 H}} H \lim _{u \rightarrow \infty}\left(m_{2}(u)\right)^{2-\frac{1}{H}}=0, \quad \nu_{2}=\lim _{u \rightarrow \infty}\left(m_{2}(u)\right)^{2} \frac{H(1-H)}{2 T^{2}}\left(\Delta_{1}(u)\right)^{2}=0, \\
y_{1,2}=\lim _{u \rightarrow \infty} m_{2}(u) \sqrt{\frac{H(1-H)}{2 T^{2}}} \Delta_{1}(u)\left(\ln m_{2}(u)\right) /\left(m_{2}(u) \Delta_{1}(u)\right)=\infty, \\
y_{2,1}=0, \quad y_{2,2}=\lim _{u \rightarrow \infty}\left(m_{2}(u)\right)^{2} \frac{H}{T} \Delta_{1}(u)\left(\ln m_{2}(u)\right)^{2} /\left(\left(m_{2}(u)\right)^{2} \Delta_{1}(u)\right)=\infty .
\end{gathered}
$$

Moreover, by Lemma $3.2, s_{u} \sim T^{\frac{1}{1-2 H}} u^{-\frac{1}{1-2 H}}$, which implies that

$$
y_{1,1}=-\lim _{u \rightarrow \infty} m_{2}(u) \sqrt{\frac{H(1-H)}{2 T^{2}}} \Delta_{1}(u) s_{u} / \Delta_{1}(u)=-Q \lim _{u \rightarrow \infty} u^{1-\frac{1}{1-2 H}}=0 .
$$


Thus by case i) of Lemma 4.1, we have that

$$
\begin{aligned}
& \mathbb{P}\left\{\sup _{(s, t) \in E_{u, 4}} Z_{u, 2}\left(s_{u}+\Delta_{1}(u) s, T-\Delta_{1}(u) t\right)>m_{2}(u)\right\} \\
& \sim\left(\mathcal{H}_{H}\right)^{2} \sqrt{\frac{2 T^{2}}{H(1-H)}} \frac{T}{H} \int_{0}^{\infty} e^{-t^{2}} d t \int_{0}^{\infty} e^{-s} d s\left(m_{2}(u)\right)^{-3}\left(\Delta_{1}(u)\right)^{-2} \Psi\left(m_{2}(u)\right) \\
& \sim 2^{-\frac{1}{H}-\frac{1}{2}} T^{3 H} \sqrt{\frac{\pi}{H^{3}(H-1)}}\left(\mathcal{H}_{H}\right)^{2} u^{\frac{2}{H}-3} \Psi\left(m_{2}(u)\right) .
\end{aligned}
$$

Inserting the above asymptotics and (26) into (25) establishes the claim. This completes the proof.

\section{Appendix}

4.1. Appendix A. This subsection is devoted to proofs of Lemmas 3.1-3.2.

Proof of Lemma 3.1: Note that for any $\delta>0$ and $u$ sufficiently large, the maximum of $\sigma_{u}^{-}(s, t)$ over $0 \leq s \leq t \leq T$ is only attained at $[0, \delta] \times[T-\delta, T]$. Next we consider the variance function $\sigma_{u}^{-}(s, t)$ over $[0, \delta] \times[T-\delta, T]$. It follows that

$$
\begin{aligned}
1-\frac{\sigma_{u}^{-}(s, t)}{\sigma_{u}^{-}(0, T)} & =1-\frac{|t-s|^{H}}{u+\mu(t-s)-\frac{1}{2}\left(t^{2 H}-s^{2 H}\right)} \frac{u+\mu T-\frac{1}{2} T^{2 H}}{T^{H}} \\
& =1-\frac{\frac{|t-s|^{H}}{T^{H}}}{\frac{u+\mu(t-s)-\frac{1}{2}\left(t^{2 H}-s^{2 H}\right)}{u+\mu T-\frac{1}{2} T^{2 H}}} \\
& =\left(1-\frac{|t-s|^{H}}{T^{H}}\right)(1+o(1))+\left(\frac{u+\mu(t-s)-\frac{1}{2}\left(t^{2 H}-s^{2 H}\right)}{u+\mu T-\frac{1}{2} T^{2 H}}-1\right)(1+o(1)) \\
& =\frac{H}{T}(T-t+s)(1+o(1))+\frac{-\mu(T-t+s)+\frac{1}{2}\left(2 H T^{2 H-1}(T-t)+s^{2 H}\right)}{u+\mu T-\frac{1}{2} T^{2 H}}(1+o(1)) \\
& =\left(\frac{H}{T}(T-t)+\frac{H}{T} s+\frac{1}{2 u} s^{2 H}\right)(1+a(\delta, u)), \quad(s, t) \in[0, \delta] \times[T-\delta, T],
\end{aligned}
$$

as $\delta$ sufficiently small and $u$ sufficiently large, where $\lim _{\delta \rightarrow 0, u \rightarrow \infty} a(\delta, u)=0$. The fact that

$$
\frac{H}{T}(T-t)+\frac{H}{T} s+\frac{1}{2 u} s^{2 H}>0
$$

for $(s, t) \in([0, \delta] \times[T-\delta, T]) \backslash\{(0, T)\}$ implies that the maximizer of $\sigma_{u}^{-}(s, t)$ over $0 \leq s \leq t \leq T$ is unique and equals $(0, T)$. This completes the proof.

Proof of Lemma 3.2: For any $\delta>0$ and $u$ sufficiently large, the maximum of $\sigma_{u}^{+}(s, t)$ over $0 \leq s \leq t \leq T$ is attained in $[0, \delta] \times[T-\delta, T]$. Next we focus on $\sigma_{u}^{+}(s, t)$ over $[0, \delta] \times[T-\delta, T]$. For $\delta>0$ sufficiently small and $u$ sufficiently large,

$$
\begin{aligned}
1-\frac{\sigma_{u}^{+}(s, t)}{\sigma_{u}^{+}(0, T)} & =1-\frac{|t-s|^{H}}{u-\mu(t-s)+\frac{1}{2}\left(t^{2 H}-s^{2 H}\right)} \frac{u-\mu T+\frac{1}{2} T^{2 H}}{T^{H}} \\
& =1-\frac{\frac{|t-s|^{H}}{T^{H}}}{\frac{u-\mu(t-s)+\frac{1}{2}\left(t^{2 H}-s^{2 H}\right)}{u-\mu T+\frac{1}{2} T^{2 H}}} \\
& =\left(1-\frac{|t-s|^{H}}{T^{H}}\right)(1+o(1))+\left(\frac{u-\mu(t-s)+\frac{1}{2}\left(t^{2 H}-s^{2 H}\right)}{u-\mu T+\frac{1}{2} T^{2 H}}-1\right)(1+o(1)) \\
& =\frac{H}{T}(T-t+s)(1+o(1))+\frac{\mu(T-t+s)-\frac{1}{2}\left(2 H T^{2 H-1}(T-t)+s^{2 H}\right)}{u-\mu T+\frac{1}{2} T^{2 H}}(1+o(1)) \\
& =\left(\frac{H}{T}(T-t)+\frac{H}{T} s\right)\left(1+a_{1}(\delta, u)\right)-\frac{1}{2 u} s^{2 H}\left(1+a_{2}(\delta, u)\right), \quad(s, t) \in[0, \delta] \times[T-\delta, T],
\end{aligned}
$$


where $\lim _{\delta \rightarrow 0, u \rightarrow \infty} a_{i}(\delta, u)=0, i=1,2$. If $H \geq \frac{1}{2}$, then

$$
1-\frac{\sigma_{u}^{+}(s, t)}{\sigma_{u}^{+}(0, T)}=\left(\frac{H}{T}(T-t)+\frac{H}{T} s\right)\left(1+a_{1}(\delta, u)\right), \quad(s, t) \in[0, \delta] \times[T-\delta, T],
$$

which implies that the maximizer of $\sigma_{u}^{+}(s, t)$ is unique and equals $(0, T)$. For $0<H<\frac{1}{2}$,

$$
\begin{aligned}
1-\frac{\sigma_{u}^{+}(s, T)}{\sigma_{u}^{+}(0, T)} & =\frac{H}{T} s\left(1+a_{1}(\delta, u)\right)-\frac{1}{2 u} s^{2 H}\left(1+a_{2}(\delta, u)\right) \\
& =\frac{H}{T} s^{2 H}\left(s^{1-2 H}\left(1+a_{1}(\delta, u)\right)-\frac{1}{2 u}\left(1+a_{2}(\delta, u)\right)\right)<0,
\end{aligned}
$$

as $s<\left(\frac{\left(1+a_{2}(\delta, u)\right)}{2 u\left(1+a_{1}(\delta, u)\right)}\right)^{\frac{1}{1-2 H}} \sim(2 u)^{-\frac{1}{1-2 H}}$. This implies that the maximum of $\sigma_{u}^{+}(s, T)$ over $[0, T]$ is attained over $(0, \delta)$ for $\delta>0$ sufficiently small and $u$ sufficiently large. We denote this point by $s_{u}$. Using the fact that

$$
\frac{\partial \sigma_{u}^{+}\left(s_{u}, T\right)}{\partial s}=\frac{-H\left(T-s_{u}\right)^{H-1}\left(u-\mu\left(T-s_{u}\right)+\frac{1}{2}\left(T^{2 H}-s_{u}^{2 H}\right)\right)-\left(T-s_{u}\right)^{H}\left(\mu-H s_{u}^{2 H-1}\right)}{\left(u-\mu\left(T-s_{u}\right)+\frac{1}{2}\left(T^{2 H}-s_{u}^{2 H}\right)\right)^{2}}=0,
$$

we have that

$$
s_{u}=\left(\frac{u}{T}+\frac{1}{2} T^{2 H-1}+\frac{\mu(1-H)}{H}+\frac{1}{2 T} s_{u}^{2 H}-\frac{\mu(1-H)}{T H} s_{u}\right)^{\frac{1}{2 H-1}} \sim T^{\frac{1}{1-2 H}} u^{-\frac{1}{1-2 H}} .
$$

Next we show that the maximizer of $\sigma_{u}^{+}(s, t)$ is $\left(s_{u}, T\right)$ for $0<H<\frac{1}{2}$ and $u$ sufficiently large. Observe that

$$
1-\frac{\sigma_{u}^{+}(s, t)}{\sigma_{u}^{+}\left(s_{u}, T\right)}=-\frac{\sigma_{u}^{+}(s, T)-\sigma_{u}^{+}\left(s_{u}, T\right)}{\sigma_{u}^{+}\left(s_{u}, T\right)}+\frac{\sigma_{u}^{+}(s, T)-\sigma_{u}^{+}(s, t)}{\sigma_{u}^{+}\left(s_{u}, T\right)} .
$$

Direct calculation gives that, as $u \rightarrow \infty$,

$$
\begin{aligned}
\sigma_{u}^{+}\left(s_{u}, T\right) & \sim \frac{T^{H}}{u}, \\
\sigma_{u}^{+}(s, T)-\sigma_{u}^{+}\left(s_{u}, T\right) & =\frac{1}{2} \frac{\partial^{2} \sigma_{u}^{+}\left(s_{u}, T\right)}{\partial^{2} s}\left(s-s_{u}\right)^{2}(1+o(1)) \sim \frac{H(H-1) T^{H-2}}{2 u}\left(s-s_{u}\right)^{2}, \\
\sigma_{u}^{+}(s, T)-\sigma_{u}^{+}(s, t) & =\frac{\partial \sigma_{u}^{+}(s, T)}{\partial t}(T-t)(1+o(1)) \sim \frac{H T^{H-1}}{u}(T-t), \quad t \rightarrow T .
\end{aligned}
$$

Thus we have

$$
1-\frac{\sigma_{u}^{+}(s, t)}{\sigma_{u}^{+}\left(s_{u}, T\right)}=\frac{H(1-H)}{2 T^{2}}\left(s-s_{u}\right)^{2}(1+o(1))+\frac{H}{T}(T-t)(1+o(1)), \quad u \rightarrow \infty,\left|s-s_{u}\right|, T-t \rightarrow 0,
$$

which implies that the maximizer of $\sigma_{u}^{+}(s, t)$ is unique and equals $\left(s_{u}, T\right)$ for $u$ large. This completes the proof.

Proof of Lemma 3.3: Recalling that $\sigma_{H}(s, t)=|t-s|^{H}$, we have, for $(s, t) \neq\left(s^{\prime}, t^{\prime}\right)$,

$$
\begin{aligned}
1 & -\operatorname{Corr}\left(B_{H}(t)-B_{H}(s), B_{H}\left(t^{\prime}\right)-B_{H}\left(s^{\prime}\right)\right) \\
& =\frac{\mathbb{E}\left\{\left(\left(B_{H}(t)-B_{H}(s)\right)-\left(B_{H}\left(t^{\prime}\right)-B_{H}\left(s^{\prime}\right)\right)\right)^{2}\right\}-\left(\sigma_{H}(s, t)-\sigma_{H}\left(s^{\prime}, t^{\prime}\right)\right)^{2}}{2 \sigma_{H}(s, t) \sigma_{H}\left(s^{\prime}, t^{\prime}\right)} \\
& =\frac{\mathbb{E}\left\{\left(\left(B_{H}(t)-B_{H}\left(t^{\prime}\right)\right)-\left(B_{H}(s)-B_{H}\left(s^{\prime}\right)\right)\right)^{2}\right\}-\left(|t-s|^{H}-\left|t^{\prime}-s^{\prime}\right|^{H}\right)^{2}}{2|t-s|^{H}\left|t^{\prime}-s^{\prime}\right|^{H}} \\
& =\frac{\left|t-t^{\prime}\right|^{2 H}+\left|s-s^{\prime}\right|^{2 H}+\left(|t-s|^{2 H}+\left|t^{\prime}-s^{\prime}\right|^{2 H}-\left|t-s^{\prime}\right|^{2 H}-\left|t^{\prime}-s\right|^{2 H}\right)-\left(|t-s|^{H}-\left|t^{\prime}-s^{\prime}\right|^{H}\right)^{2}}{2|t-s|^{H}\left|t^{\prime}-s^{\prime}\right|^{H}} .
\end{aligned}
$$

Using Taylor formula, we have that for $(s, t) \in\left[0, \delta_{u}\right] \times\left[T-\delta_{u}, T\right]$, with $\lim _{u \rightarrow \infty} \delta_{u}=0$ and $u$ sufficiently large

$$
\begin{aligned}
|t-s|^{2 H}-\left|t-s^{\prime}\right|^{2 H}-\left(\left|t^{\prime}-s\right|^{2 H}-\left|t^{\prime}-s^{\prime}\right|^{2 H}\right) & =2 H\left(\left|\theta_{1}-s\right|^{2 H-1}-\left|\theta_{1}-s^{\prime}\right|^{2 H-1}\right)\left(t-t^{\prime}\right) \\
& =2 H(2 H-1)\left(\theta_{1}-\theta_{2}\right)^{2 H-2}\left(s-s^{\prime}\right)\left(t-t^{\prime}\right), \\
\left(|t-s|^{H}-\left|t^{\prime}-s^{\prime}\right|^{H}\right)^{2} & =\left(H \theta_{3}\left(t-t^{\prime}-s+s^{\prime}\right)\right)^{2},
\end{aligned}
$$


where $\theta_{1} \in\left(t, t^{\prime}\right), \theta_{2} \in\left(s, s^{\prime}\right)$ and $\theta_{3} \in\left(t-s, t^{\prime}-s^{\prime}\right)$. Moreover,

$$
\lim _{u \rightarrow \infty} \lim _{s, t \in\left[0, \delta_{u}\right] \times\left[T-\delta_{u}, T\right]}|| t-\left.s\right|^{H}-T^{H} \mid=0 .
$$

Consequently, for $\lim _{u \rightarrow \infty} \delta_{u}=0$,

$$
\lim _{u \rightarrow \infty} \sup _{(s, t) \neq\left(s^{\prime}, t^{\prime}\right),(s, t),\left(s^{\prime}, t^{\prime}\right) \in\left[0, \delta_{u}\right] \times\left[T-\delta_{u}, T\right]}\left|\frac{1-\operatorname{Corr}\left(B_{H}(t)-B_{H}(s), B_{H}\left(t^{\prime}\right)-B_{H}\left(s^{\prime}\right)\right)}{\frac{\left|s-s^{\prime}\right|^{2 H}+\left|t-t^{\prime}\right|^{2 H}}{2 T^{2 H}}}-1\right|=0 .
$$

4.2. Appendix B. In this subsection we present some useful results derived in [17]. First, we give an adaptation of Theorem 3.2 in [17] to our setting. Let $X_{u}(s, t),(s, t) \in \prod_{i=1,2}\left[a_{i}(u), b_{i}(u)\right]$ with $(0,0) \in \prod_{i=1,2}\left[a_{i}(u), b_{i}(u)\right]$, be a family of centered continuous Gaussian random fields with variance function $\sigma_{u}(s, t)$ satisfying

$$
\sigma_{u}(0,0)=1, \quad \lim _{u \rightarrow \infty} \sup _{(s, t) \neq(0,0),(s, t) \in \prod_{i=1,2}\left[a_{i}(u), b_{i}(u)\right]}\left|\frac{1-\sigma_{u}(s, t)}{\frac{|s|^{\beta_{1}}}{g_{1}(u)}+\frac{|t|^{\beta_{2}}}{g_{2}(u)}}-1\right|=0
$$

where $\beta_{i}>0, i=1,2, \lim _{u \rightarrow \infty} g_{i}(u)=\infty, i=1,2, \lim _{u \rightarrow \infty} \frac{\left|a_{i}(u)\right|^{\beta_{1}}}{g_{1}(u)}+\frac{+\left|b_{i}(u)\right|^{\beta_{2}}}{g_{2}(u)}=0, i=1,2$, and correlation function satisfying

$$
\lim _{u \rightarrow \infty} \sup _{(s, t) \neq\left(s^{\prime}, t^{\prime}\right),(s, t),\left(s^{\prime}, t^{\prime}\right) \in \prod_{i=1,2}\left[a_{i}(u), b_{i}(u)\right]}\left|n^{2}(u) \frac{1-\operatorname{Corr}\left(X_{u}(s, t), X_{u}\left(s^{\prime}, t^{\prime}\right)\right)}{\left|s-s^{\prime}\right|^{\alpha}+\left|t-t^{\prime}\right|^{\alpha}}-1\right|=0,
$$

with $\alpha \in(0,2]$ and $\lim _{u \rightarrow \infty} n(u)=\infty$.

We suppose that $\lim _{u \rightarrow \infty} \frac{n^{2}(u)}{g_{i}(u)}=\nu_{i} \in[0, \infty], i=1,2$.

Lemma 4.1. Let $X_{u}(s, t),(s, t) \in \prod_{i=1,2}\left[a_{i}(u), b_{i}(u)\right]$ with $(0,0) \in \prod_{i=1,2}\left[a_{i}(u), b_{i}(u)\right]$ be a family of centered continuous Gaussian random fields satisfying (27) and (28).

i) If $\nu_{i}=0, i=1,2$ and for $i=1,2$,

$$
\lim _{u \rightarrow \infty} \frac{(n(u))^{2 / \beta_{i}} a_{i}(u)}{\left(g_{i}(u)\right)^{1 / \beta_{i}}}=y_{i, 1}, \quad \lim _{u \rightarrow \infty} \frac{(n(u))^{2 / \beta_{i}} b_{i}(u)}{\left(g_{i}(u)\right)^{1 / \beta_{i}}}=y_{i, 2}, \quad \lim _{u \rightarrow \infty} \frac{(n(u))^{2 / \beta_{i}}\left(a_{i}^{2}(u)+b_{i}^{2}(u)\right)}{\left(g_{i}(u)\right)^{2 / \beta_{i}}}=0,
$$

with $-\infty \leq y_{i, 1}<y_{i, 2} \leq \infty$, then

$$
\mathbb{P}\left\{\sup _{(s, t) \in \prod_{i=1,2}\left[a_{i}(u), b_{i}(u)\right]} X_{u}(s, t)>n(u)\right\} \sim\left(\mathcal{H}_{\alpha / 2}\right)^{2} \prod_{i=1}^{2} \int_{y_{i, 1}}^{y_{i, 2}} e^{-|s|^{\beta_{i}}} d s \prod_{i=1}^{2}\left(\frac{g_{i}(u)}{n^{2}(u)}\right)^{1 / \beta_{i}} \Psi(n(u)) .
$$

ii) If $\nu_{i} \in(0, \infty)$ and further $\lim _{u \rightarrow \infty} a_{i}(u)=a_{i} \in[-\infty, 0], \lim _{u \rightarrow \infty} b_{i}(u)=b_{i} \in[0, \infty]$, then

$$
\mathbb{P}\left\{\sup _{(s, t) \in \prod_{i=1,2}\left[a_{i}(u), b_{i}(u)\right]} X_{u}(s, t)>n(u)\right\} \sim \prod_{i=1}^{2} \mathcal{P}_{\alpha / 2}^{v_{i}, \beta_{i}}\left(\left[a_{i}, b_{i}\right]\right) \Psi(n(u)),
$$

where

$$
\mathcal{P}_{\alpha / 2}^{v_{i}, \beta_{i}}\left(\left[a_{i}, b_{i}\right]\right)=\mathbb{E}\left\{\sup _{t \in\left[a_{i}, b_{i}\right]} e^{\sqrt{2} B_{\alpha / 2}(t)-|t|^{\alpha}-\nu_{i}|t|^{\beta_{i}}}\right\} \in(0, \infty), i=1,2 .
$$

iii) If $\nu_{i}=\infty, i=1,2$, then

$$
\mathbb{P}\left\{\sup _{(s, t) \in \prod_{i=1,2}\left[a_{i}(u), b_{i}(u)\right]} X_{u}(s, t)>n(u)\right\} \sim \Psi(n(u)) .
$$

Next we give a simpler version of Proposition 2.2 in [17]. Denote by $\Lambda(u)$ a series of index sets depending on $u$ and by $\left[a_{1}, a_{2}\right] \times\left[b_{1}, b_{2}\right]$ a rectangle with $a_{1}<a_{2}$ and $b_{1}<b_{2}$. Let $X_{u, k, l}(s, t),(s, t) \in\left[a_{1}, a_{2}\right] \times\left[b_{1}, b_{2}\right],(k, l) \in \Lambda(u)$ be a family of two-dimensional continuous Gaussian random fields with mean 0 and variance function 1 . There exist $n_{k, l}(u),(k, l) \in \Lambda(u)$ satisfying

$$
\lim _{u \rightarrow \infty} \sup _{(k, l),\left(k^{\prime}, l^{\prime}\right) \in \Lambda(u)}\left|\frac{n_{k, l}(u)}{n_{k^{\prime} l^{\prime}}(u)}-1\right|=0, \quad \lim _{u \rightarrow \infty} \inf _{(k, l) \in \Lambda(u)} n_{k, l}=\infty,
$$


such that the correlation function satisfies

$(30) \lim _{u \rightarrow \infty} \sup _{(k, l) \in \Lambda(u)} \sup _{(s, t) \neq\left(s^{\prime}, t^{\prime}\right),(s, t),\left(s^{\prime}, t^{\prime}\right) \in\left[a_{1}, a_{2}\right] \times\left[b_{1}, b_{2}\right]}\left|\left(n_{k, l}(u)\right)^{2} \frac{1-\operatorname{Corr}\left(X_{u, k, l}(s, t), X_{u, k, l}\left(s^{\prime}, t^{\prime}\right)\right)}{\left|s-s^{\prime}\right|^{\alpha_{1}}+\left|t-t^{\prime}\right|^{\alpha_{2}}}-1\right|=0$,

where $\alpha_{i} \in(0,2], i=1,2$.

Then Proposition 2.2 in [17] leads to the following result.

Lemma 4.2. Let $X_{u, k, l}(s, t),(s, t) \in E,(k, l) \in \Lambda(u)$ be a family of centered two-dimensional continuous Gaussian random fields with unit variance. Assume further that (29)-(30) hold. Then

$$
\lim _{u \rightarrow \infty} \sup _{(k, l) \in \Lambda(u)}\left|\frac{\mathbb{P}\left\{\sup _{(s, t) \in\left[a_{1}, a_{2}\right] \times\left[b_{1}, b_{2}\right]} X_{u, k, l}(s, t)>n_{k, l}(u)\right\}}{\Psi\left(n_{k, l}(u)\right)}-\mathcal{H}_{\frac{\alpha_{1}}{2}}\left(\left[a_{1}, a_{2}\right]\right) \mathcal{H}_{\frac{\alpha_{1}}{2}}\left(\left[b_{1}, b_{2}\right]\right)\right|=0 .
$$

Finally, we display a lemma concerning the uniform double maximum, a simpler version of Corollary 3.2 in [17]. Let $E_{u}$ be a family of non-empty compact subset of $\mathbb{R}^{2}$ and $A_{i} \subset[0, S]^{2}, i=1,2$ be two non-empty compact subsets of $\mathbb{R}^{2}$. Denote by $\Lambda_{0}(u)=\left\{\left(k_{1}, l_{1}, k_{2}, l_{2}\right):\left(k_{i}, l_{i}\right)+A_{i} \subset E_{u}, i=1,2\right\}$. Let $n(u)$ and $n_{k_{i}, l_{i}}(u),\left(k_{i}, l_{i}\right)+A_{i} \subset E_{u}$ be a family of positive functions such that

$$
\lim _{u \rightarrow \infty} \sup _{\left(k_{i}, l_{i}\right)+A_{i} \in E_{u}}\left|\frac{n_{k_{i}, l_{i}}(u)}{n(u)}-1\right|=0, \quad i=1,2, \quad \lim _{u \rightarrow \infty} n(u)=\infty .
$$

Lemma 4.3. Let $X_{u}(s, t),(s, t) \in E_{u}$ be a family of centered Gaussian random fields with variance 1 and correlation function satisfying

$$
\lim _{u \rightarrow \infty} \sup _{(s, t) \neq\left(s^{\prime}, t^{\prime}\right),(s, t),\left(s^{\prime}, t^{\prime}\right) \in E_{u}}\left|(n(u))^{2} \frac{1-\operatorname{Corr}\left(X_{u}(s, t), X_{u}\left(s^{\prime}, t^{\prime}\right)\right)}{\left|s-s^{\prime}\right|^{\alpha_{1}}+\left|t-t^{\prime}\right|^{\alpha_{2}}}-1\right|=0 .
$$

Moreover, there exists $\delta>0$ such that for $u$ large enough

$$
\operatorname{Corr}\left(X_{u}(s, t), X_{u}\left(s^{\prime}, t^{\prime}\right)\right)>\delta-1,(s, t),\left(s^{\prime}, t^{\prime}\right) \in E_{u} .
$$

If further (31) is satisfied, then there exit $\mathcal{C}, \mathcal{C}_{1}>0$ such that for all $u$ large

$\sup _{\left(k_{1}, l_{1}, k_{2}, l_{2}\right) \in \Lambda_{0}(u), A_{i} \subset[0, S]^{2}, A_{i} \neq \emptyset, i=1,2} \frac{\mathbb{P}\left\{\sup _{(s, t) \in\left(k_{1}, l_{1}\right)+A_{1}} X_{u}(s, t)>n_{k_{1}, l_{1}}(u), \sup _{(s, t) \in\left(k_{2}, l_{2}\right)+A_{2}} X_{u}(s, t)>n_{k_{2}, l_{2}}(u)\right\}}{e^{-\mathcal{C}_{1}\left(F\left(\left(k_{1}, l_{1}\right)+A_{1},\left(k_{2}, l_{2}\right)+A_{2}\right)\right)^{\frac{1}{2} \min \left(\alpha_{1}, \alpha_{2}\right)}} S^{4} \Psi\left(n_{k_{1}, l_{1}, k_{2}, l_{2}}(u)\right)} \leq \mathcal{C}$,

where

$$
F(A, B)=\inf _{s \in A, t \in B}\|s-t\|, \quad n_{k_{1}, l_{1}, k_{2}, l_{2}}(u)=\min \left(n_{k_{1}, l_{1}}(u), n_{k_{2}, l_{2}}(u)\right),
$$

and $\mathcal{C}$ and $\mathcal{C}_{1}$ are independent of $u$ and $S$.

\section{Acknowledgments}

The authors thank the referee for his/her useful suggestions and detailed comments which significantly improved the readability of this contribution. We also sincerely thank Professor Enkelejd Hashorva for his encouragement and support to finish this work. Thanks to the Swiss National Science Foundation Grant 200021-175752/1.

\section{REFERENCES}

[1] O. Hadjiliadis and J. Večer, "Drawdowns preceding rallies in the Brownian motion model," Quant. Finance, vol. 6, no. 5, pp. 403-409, 2006.

[2] H. Zhang, T. Leung, and O. Hadjiliadis, "Stochastic modeling and fair valuation of drawdown insurance," Insurance Math. Econom., vol. 53, no. 3, pp. 840-850, 2013.

[3] J. James and L. Yang, "Stop-losses, maximum drawdown-at-risk and replicating financial time series with the stationary bootstrap," Quant. Finance, vol. 10, no. 1, pp. 1-12, 2010.

[4] L. Pospisil and J. Vecer, "Portfolio sensitivity to changes in the maximum and the maximum drawdown," Quant. Finance, vol. 10, no. 6, pp. 617-627, 2010. 
[5] V. Cherny and J. Obł ój, "Portfolio optimisation under non-linear drawdown constraints in a semimartingale financial model," Finance Stoch., vol. 17, no. 4, pp. 771-800, 2013.

[6] C. Kardaras, J. Obł ój, and E. Platen, "The numéraire property and long-term growth optimality for drawdown-constrained investments," Math. Finance, vol. 27, no. 1, pp. 68-95, 2017.

[7] M. Mandjes, Large deviations for Gaussian queues. John Wiley \& Sons, Ltd., Chichester, 2007. Modelling communication networks.

[8] K. Dębicki and M. Mandjes, Queues and Lévy fluctuation theory. Universitext, Springer, Cham, 2015.

[9] E. J. Baurdoux, Z. Palmowski, and M. R. Pistorius, "On future drawdowns of Lévy processes," Stochastic Process. Appl., vol. 127, no. 8, pp. 2679-2698, 2017.

[10] D. Landriault, B. Li, and H. Zhang, "On magnitude, asymptotics and duration of drawdowns for Lévy models," Bernoulli, vol. 23, no. 1, pp. 432-458, 2017.

[11] L. Pospisil, J. Vecer, and O. Hadjiliadis, "Formulas for stopped diffusion processes with stopping times based on drawdowns and drawups," Stochastic Process. Appl., vol. 119, no. 8, pp. 2563-2578, 2009.

[12] R. J. Elliott and J. van der Hoek, "A general fractional white noise theory and applications to finance," Math. Finance, vol. 13, no. 2, pp. 301-330, 2003.

[13] K. Shokrollahi and A. Kiliçman, "The valuation of currency options by fractional Brownian motion," SpringerPlus, no. 5, p. 1145, 2016.

[14] R. Douady, A. N. Shiryaev, and M. Yor, "On the probability characteristics of 'drop' variables in standard Brownian motion," Theory Probab. Appl., vol. 44, no. 1, pp. 29-38, 2000.

[15] M. Magdon-Ismail, A. F. Atiya, A. Pratap, and Y. S. Abu-Mostafa, "On the maximum drawdown of a Brownian motion," J. Appl. Probab., vol. 41, no. 1, pp. 147-161, 2004.

[16] P. Salminen and P. Vallois, "On maximum increase and decrease of Brownian motion," Ann. Inst. H. Poincaré Probab. Statist., vol. 43, no. 6, pp. 655-676, 2007.

[17] K. Dȩbicki, E. Hashorva, and P. Liu, "Uniform tail approximation of homogenous functionals of Gaussian fields," Adv. in Appl. Probab., vol. 49, pp. 1037-1066, 2017.

[18] V. Piterbarg, Asymptotic methods in the theory of Gaussian processes and fields, vol. 148 of Translations of Mathematical Monographs. Providence, RI: American Mathematical Society, 1996.

[19] V. Piterbarg, "Large deviations of a storage process with fractional Browanian motion as input," Extremes, vol. 4, pp. 147-164, 2001.

[20] E. Hashorva, L. Ji, and V. I. Piterbarg, "On the supremum of $\gamma$-reflected processes with fractional Brownian motion as input," Stochastic Process. Appl., vol. 123, no. 11, pp. 4111-4127, 2013.

[21] K. Dębicki and P. Liu, "Extremes of stationary Gaussian storage models," Extremes, vol. 19, no. 2, pp. 273302, 2016.

[22] K. Dębicki, E. Hashorva, and P. Liu, "Extremes of $\gamma$-reflected Gaussian process with stationary increments," ESAIM Probab. Stat., vol. 21, pp. 495-535, 2017.

[23] R. Adler and J. Taylor, Random fields and geometry. Springer Monographs in Mathematics, New York: Springer, 2007.

[24] K. Dębicki, "Ruin probability for Gaussian integrated processes," Stochastic Process. Appl., vol. 98, no. 1, pp. 151-174, 2002.

[25] J. Hüsler and V. I. Piterbarg, "On the ruin probability for physical fractional Brownian motion," Stochastic Process. Appl., vol. 113, no. 2, pp. 315-332, 2004.

[26] A. Dieker, "Extremes of Gaussian processes over an infinite horizon," Stochastic Process. Appl., vol. 115, no. 2, pp. 207-248, 2005. 
[27] K. Dȩbicki, S. Engelke, and E. Hashorva, "Generalized Pickands constants and stationary max-stable processes," Extremes, vol. 20, no. 3, pp. 493-517, 2017.

[28] K. Dębicki, Z. Michna, and T. Rolski, "Simulation of the asymptotic constant in some fluid models," Stoch. Models, vol. 19, no. 3, pp. 407-423, 2003.

[29] A. Dieker and B. Yakir, "On asymptotic constants in the theory of Gaussian processes," Bernoulli, vol. 20, no. 3, pp. 1600-1619, 2014.

[30] A. B. Dieker and T. Mikosch, "Exact simulation of Brown-Resnick random fields at a finite number of locations," Extremes, vol. 18, no. 2, pp. 301-314, 2015.

[31] L. Bai, K. Dębicki, E. Hashorva, and L. Luo, "On generalised Piterbarg constants," Methodol. Comput. Appl. Probab., 2017.

[32] A. J. Harper, "Pickands' constant $H_{\alpha}$ does not equal $1 / \Gamma(1 / \alpha)$, for small $\alpha$," Bernoulli, vol. 23, no. 1, pp. 582-602, 2017.

[33] K. Dębicki and K. M. Kosiński, "An Erdös-Révész type law of the iterated logarithm for reflected fractional Brownian motion," Extremes, vol. 20, no. 4, pp. 729-749, 2017.

[34] K. M. Kosiński and P. Liu, "Sample path properties of reflected Gaussian processes," ALEA Lat. Am. J. Probab. Math. Stat., vol. 15, pp. 453-478, 2018.

Long Bai, Department of Actuarial Science, University of Lausanne, Unil-Dorigny, 1015 Lausanne, Switzerland

E-mail address: Long.Bai@unil.ch

Liu, Peng, Department of Actuarial Science, University of Lausanne, Unil-Dorigny, 1015 Lausanne, Switzerland

E-mail address: peng.liu@unil.ch 